BULLETIN (New Series) OF THE

AMERICAN MATHEMATICAL SOCIETY

Volume 40, Number 4, Pages 479-519

S 0273-0979(03)00988-1

Article electronically published on July 10, 2003

\title{
EVOLUTIONARY GAME DYNAMICS
}

\author{
JOSEF HOFBAUER AND KARL SIGMUND
}

\begin{abstract}
Evolutionary game dynamics is the application of population dynamical methods to game theory. It has been introduced by evolutionary biologists, anticipated in part by classical game theorists. In this survey, we present an overview of the many brands of deterministic dynamical systems motivated by evolutionary game theory, including ordinary differential equations (and, in particular, the replicator equation), differential inclusions (the best response dynamics), difference equations (as, for instance, fictitious play) and reaction-diffusion systems. A recurrent theme (the so-called 'folk theorem of evolutionary game theory') is the close connection of the dynamical approach with the Nash equilibrium, but we show that a static, equilibriumbased viewpoint is, on principle, unable to always account for the long-term behaviour of players adjusting their behaviour to maximise their payoff.
\end{abstract}

\section{INTRODUCTION}

According to the eminent biologist Ernst Mayr, the greatest conceptual revolution that has taken place in biology was the replacement of typological thinking by population thinking Mayr70. A similar conceptual revolution has occurred in game theory. It was fostered, appropriately, by evolutionary biologists such as William D. Hamilton and John Maynard Smith. The resulting population-based, 'evolutionary' game theory has found many applications in non-biological fields like economics or learning theory and presents an important enrichment of 'classical' game theory, which is centered on the concept of a rational individual.

This survey focuses on the mathematical core of evolutionary game theory and concentrates on deterministic evolutionary game dynamics, a dynamics which describes how the frequencies of strategies within a population change in time, according to the strategies' success. This requires certain modifications in the basic conceptual approach. At the risk of over-simplification, one can say that classical game theory deals with a rational individual, or 'player', who is engaged in a given interaction or 'game' with other players and has to decide between different options, or 'strategies', in order to maximise a 'payoff' which depends on the strategies of the co-players (who, in turn, attempt to maximise their payoff). In contrast, evolutionary game theory deals with entire populations of players, all programmed to use some strategy (or type of behaviour). Strategies with high payoff will spread within the population (this can be achieved by learning, by copying or inheriting strategies, or even by infection). The payoffs depend on the actions of the coplayers and hence on the frequencies of the strategies within the population. Since

Received by the editors March 7, 2003, and, in revised form, April 12, 2003.

2000 Mathematics Subject Classification. Primary 91A22; Secondary 91-02, 92-02, 34D20. 
these frequencies change according to the payoffs, this yields a feedback loop. The dynamics of this feedback loop is the object of evolutionary game theory.

This ansatz may well be what Oskar Morgenstern and John von Neumann had in mind when, in the introduction of their classical treatise on game theory [NM47, they underlined the desirability of a 'dynamic' approach to complement their 'static' solution concept, and certainly John Nash had anticipated nothing else when (in an unpublished section of his thesis [Na51]) he sketched a 'mass action approach' to his equilibrium notion which, many years later, was re-discovered as the evolutionary approach (see [Le94, [Na96, p.32], or [Na01]).

The feedback dynamics depend strongly, of course, on the population structure, on the underlying game and on the way strategies spread. Thus there are many 'game dynamics', which can be discrete or continuous, stochastic or deterministic. We shall divide this expository survey into three parts. Section 2 deals with the 'replicator dynamics': it starts with a 'folk theorem' connecting the dynamics with Nash equilibria, offers some results on a classification of its long-term behaviour, discusses the notion of permanence (where no strategy gets eliminated), and investigates the concept of an evolutionarily stable equilibrium, before turning to bimatrix games. Section 3 deals with 'other game dynamics': these include imitation dynamics, the best response dynamics, smoothed best reply and the Brown-von NeumannNash dynamics. Among the recurrent questions are whether variants of the 'folk theorem' remain valid, and whether dominated strategies get eliminated. A central result on general 'adjustment dynamics' shows that every reasonable adaptation process will fail, for some games, to lead to a Nash equilibrium. Section 4 deals with 'extensions and applications', including, for instance, discrete time dynamics, or models based on diffusion in spatially distributed populations. This section covers methods used in evolutionary biology, as e.g. population genetics and adaptive dynamics, as well as approaches from classical, rationality-based game theory, as for instance the technique of fictitious play. We conclude by stressing the close links of evolutionary game dynamics with Nash's original proofs of his equilibrium theorem.

The emphasis on replicator dynamics in this survey is not meant to suggest that it is as important as all other dynamics together, but it serves conveniently for expository purposes and reflects some of the history of the subject. It is not possible to present here a complete overview of the whole area of evolutionary games - for this, the format of book-length treatments like [MS82, HoS88], [BoP89], Cr92, We95], [V96], Sa97], FL98], HoSi98, [Y98, Gi00] and [Cr03] is much more appropriate; what we attempt is a signposted introduction aimed at mathematicians sensitive to the charms of new and variegated deterministic dynamics arising out of simple, individual-based models of social evolution. For previous surveys directed to biologists and economists, see [Hi87], HaS94], Ka97], [Mai98, [Bö00]. For the connection of evolutionary game theory with classical game theory, we refer to We95] and vD91] and for a very recent full-length treatment of extensive form games, to $\mathrm{Cr} 03$.

\section{REPLiCATOR DYNAMICS}

2.1 Nash equilibria. The simplest type of game has only two players, $I$ and $I I$, each with a finite set of options or pure strategies, Strat (I) resp. Strat $(I I)$. (The even simpler case of a one-player game reduces to an optimisation problem.) 
We shall denote by $a_{i j}$ resp. $b_{i j}$ the payoff (or, if this is a random variable, its expected value) for player $I$ resp. $I I$ when $I$ uses strategy $i \in S t r a t(I)$ and $I I$ uses $j \in \operatorname{Strat}(I I)$. Thus the payoffs are given by the $n \times m$-matrices $A$ and $B$, with $n$ and $m$ as the cardinalities of the sets of pure strategies.

The mixed strategy of player $I$ which consists in using $i \in \operatorname{Strat}(I)$ with probability $x_{i}$ will be denoted by the (column) vector $\mathbf{x}=\left(x_{1}, \ldots, x_{n}\right)^{T}$, which is an element of the unit simplex $S_{n}$ spanned by the vectors $\mathbf{e}_{i}$ of the standard unit base: these vectors will be identified with the elements of $\operatorname{Strat}(I)$. Similarly, the unit simplex $S_{m}$ spanned by the vectors $\mathbf{f}_{j}$ corresponds to the set of mixed strategies for player $I I$. If player $I$ uses $\mathbf{x} \in S_{n}$ and $I I$ uses $\mathbf{y} \in S_{m}$, then the former has as his expected payoff $\mathbf{x}^{T} A \mathbf{y}$ and the latter $\mathbf{x}^{T} B \mathbf{y}$. The strategy $\mathbf{x} \in S_{n}$ is said to be a best reply to $\mathbf{y} \in S_{m}$ if

$$
\mathbf{z}^{T} A \mathbf{y} \leq \mathbf{x}^{T} A \mathbf{y}
$$

for all $\mathbf{z} \in S_{n}$. The (compact, convex, non-empty) set of all best replies to $\mathbf{y}$ is denoted by $B R(\mathbf{y})$. A pair $(\mathbf{x}, \mathbf{y}) \in S_{n} \times S_{m}$ is a Nash equilibrium (NE) if $\mathbf{x} \in B R(\mathbf{y})$ and (with an obvious abuse of notation) $\mathbf{y} \in B R(\mathbf{x})$. As we shall presently see, a simple fixed-point argument shows that such NE always exist. The pair is said to be a strict Nash equilibrium if $\mathbf{x}$ is the unique best reply to $\mathbf{y}$ and vice versa. Necessarily, strict NE are of the form $\left(\mathbf{e}_{i}, \mathbf{f}_{j}\right)$. If two strategies form a NE, none of the players has an incentive to deviate unilaterally. In this sense, such an outcome satisfies a consistency condition.

In order to transfer this to a population setting, it is convenient to restrict attention, to begin with, to the case where the two players $I$ and $I I$ are interchangeable individuals within the population, i.e. to consider only the case where the two players do not appear in different roles - as, for instance, buyer and seller - but have the same strategy set and the same payoff matrix. More precisely, we shall first consider symmetric games, defined by $\operatorname{Strat}(I)=\operatorname{Strat}(I I)$ and $A=B^{T}$. For symmetric games, players cannot be distinguished and only symmetric pairs $(\mathbf{x}, \mathbf{x})$ of strategies are of interest. We shall therefore say, by abuse of language, that strategy $\mathbf{x} \in S_{n}$ is a Nash equilibrium if

$$
\mathbf{z}^{T} A \mathbf{x} \leq \mathbf{x}^{T} A \mathbf{x}
$$

for all $\mathbf{z} \in S_{n}$, i.e. if $\mathbf{x}$ is a best reply to itself. The equilibrium is said to be strict if equality holds only for $\mathbf{z}=\mathbf{x}$.

2.2 The replicator equation. Let us consider now a population consisting of $n$ types, and let $x_{i}$ be the frequency of type $i$. Then the state of the population is given by $\mathrm{x} \in S_{n}$. We shall now assume that the $x_{i}$ are differentiable functions of time $t$ (which requires assuming that the population is infinitely large or that the $x_{i}$ are expected values for an ensemble of populations) and postulate a law of motion for $\mathbf{x}(t)$. If individuals meet randomly and then engage in a symmetric game with payoff matrix $A$, then $(A \mathbf{x})_{i}$ is the expected payoff for an individual of type $i$ and $\mathbf{x}^{T} A \mathbf{x}$ is the average payoff in the population state $\mathbf{x}$. Let us assume that the per capita rate of growth, i.e. the logarithmic derivative $\left(\log x_{i}\right){ }^{\cdot}=\dot{x}_{i} / x_{i}$, is given by the difference between the payoff for type $i$ and the average payoff in the population. This yields the replicator equation

$$
\dot{x}_{i}=x_{i}\left((A \mathbf{x})_{i}-\mathbf{x}^{T} A \mathbf{x}\right)
$$


for $i=1, \ldots, n$. The replicator equation, which was introduced in TaJ78] and baptised in [ScS83], describes a selection process: more successful strategies spread in the population. (This differential equation appeared earlier in different contexts such as population genetics and chemical networks, see e.g. HoS88 or HoSi98 for historical remarks.)

Since the hyperplanes $\sum x_{i}=1$ and $x_{i}=0$ are invariant, it follows that the unit simplex $S_{n}$ is invariant, and from now on we shall consider only the restriction of (3) to $S_{n}$, the state space of the population. The boundary faces

$$
S_{n}(J)=\left\{\mathbf{x} \in S_{n}: x_{i}=0 \quad \text { for all } i \in J\right\}
$$

(where $J$ is any non-trivial subset of $\{1, \ldots, n\}$ ) are also invariant under (3), and so is the interior, int $S_{n}$, of the state space, where $x_{i}>0$ for all $i$. Two simple facts will be frequently used:

(a) adding a constant $c_{j}$ to all entries in the $j$-th column of $A$ does not affect the replicator equation;

(b) whenever the power product $P=\prod_{i} x_{i}^{\alpha_{i}}$ is defined, its time-derivative satisfies

$$
\dot{P}=P \sum \alpha_{i}\left[(A \mathbf{x})_{i}-\mathbf{x}^{T} A \mathbf{x}\right] .
$$

In order to describe the long-term behaviour of the dynamics, we shall say that a rest point $\mathbf{z}$ is stable if for every neighborhood $U$ of $\mathbf{z}$ there exists a neighborhood $V$ of $\mathbf{z}$ such that $\mathbf{x} \in V$ implies $\mathbf{x}(t) \in U$ for all $t \geq 0$. The rest point $\mathbf{z}$ is said to be attracting if it has a neighborhood $U$ such that $\mathbf{x}(t) \rightarrow \mathbf{z}$ for $t \rightarrow+\infty$ holds for all $\mathbf{x} \in U$. It is asymptotically stable (or an attractor) if it is both stable and attracting, and globally stable if it is stable and $\mathbf{x}(t) \rightarrow \mathbf{z}$ for $t \rightarrow+\infty$ whenever $x_{i}>0$ for all $i$ with $z_{i}>0$. (One cannot request convergence for all $\mathbf{x} \in S_{n}$ since boundary faces are invariant.) Similar definitions are used if $\mathbf{z}$ is replaced by a closed set of rest points, or a compact invariant set.

2.3 Nash equilibria and the replicator equation. The rest points of the replicator equation, i.e. the zeros of the vector field given by the right hand side of (3), are the points $\mathbf{x} \in S_{n}$ satisfying $(A \mathbf{x})_{i}=\mathbf{x}^{T} A \mathbf{x}$ for all $i \in \operatorname{supp}(\mathbf{x})$. Thus a rest point in int $S_{n}$ (an interior rest point) is a solution of the system of linear equations $(A \mathbf{x})_{1}=\cdots=(A \mathbf{x})_{n}$ (generically, there exists at most one such solution), and the rest points in the interior of each subface $S_{n}(J)$ are obtained similarly. In particular, the corners $\mathbf{e}_{i}$ of the state simplex are always rest points.

There is a close relation between the rest points of the replicator equation and the Nash equilibria given by the (symmetric) game with payoff matrix $A$. Indeed, it is easy to see (see, for instance, Bo86], [Nac90], or [We95], [HoSi98]) that

(a) if $\mathbf{z}$ is a Nash equilibrium, then it is a rest point;

(b) if $\mathbf{z}$ is a strict Nash equilibrium, then it is asymptotically stable;

(c) if the rest point $\mathbf{z}$ is the limit of an interior orbit (an orbit $\mathbf{x}(t)$ in int $S_{n}$ ) for $t \rightarrow+\infty$, then $\mathbf{z}$ is a Nash equilibrium; and

(d) if the rest point $\mathbf{z}$ is stable, then it is a Nash equilibrium.

This is sometimes referred to as the folk theorem of evolutionary game theory (cf. Cr03]). None of the converse statements holds. Trivially, every interior rest point is a Nash equilibrium. At a boundary rest point $\mathbf{z}$, the difference $(A \mathbf{z})_{i}-\mathbf{z}^{T} A \mathbf{z}$ is an eigenvalue for the Jacobian of the replicator equation whose eigenvector is transversal to the face $z_{i}=0$. Hence a rest point $\mathbf{z}$ is a Nash equilibrium iff all 
its transversal eigenvalues are nonpositive. This yields a proof for the existence of Nash equilibria in terms of population dynamics:

Theorem 1. Each game has at least one Nash equilibrium.

Indeed, the equation

$$
\dot{x}_{i}=x_{i}\left((A \mathbf{x})_{i}-\mathbf{x}^{T} A \mathbf{x}-n \varepsilon\right)+\varepsilon
$$

is a perturbation of the replicator equation (3) with a small $\varepsilon>0$ representing a constant immigration term. This equation maintains the relation $\sum_{i} \dot{x}_{i}=0$ on $S_{n}$ and the flow on the boundary points into the interior of $S_{n}$. By a variant of Brouwer's fixed point theorem, there exists at least one rest point $\mathbf{z}(\varepsilon)$ in int $S_{n}$, and

$$
(A \mathbf{z}(\varepsilon))_{i}-\mathbf{z}(\varepsilon)^{T} A \mathbf{z}(\varepsilon)-n \varepsilon=-\frac{\varepsilon}{z_{i}(\varepsilon)}<0 .
$$

Any accumulation point $\mathbf{z}$ of $\mathbf{z}(\varepsilon)$ (for $\varepsilon \rightarrow 0$ ) is an NE.

A simple modification of this argument (see [HoS88], HoSi98]) shows that if all NE are regular (i.e., with non-singular Jacobian), then their number must be odd, as shown earlier e.g. in Har73.

2.4 Classification of phase portraits. We consider two replicator equations as equivalent if there exists a homeomorphism of $S_{n}$ mapping the (oriented) orbits of one equation onto those of the other. The task of classifying the equivalence classes is solved only in low dimensions.

For $n=2$ the replicator dynamics reduces (with $x=x_{1}$ and $1-x=x_{2}$ ) to the equation

$$
\dot{x}=x(1-x)\left((A \mathbf{x})_{1}-(A \mathbf{x})_{2}\right)
$$

on $[0,1]$ which admits only three outcomes (apart from the trivial case that all points are rest points): either there is no interior equilibrium, in which case one or the other frequency converges to 0 (the corresponding strategy, or type, is said to be dominated by the other), or else there exists an interior rest point. If this point is (globally) stable, it is the only (symmetric) NE and the outcome is a stable coexistence of both types. If it is unstable, the two pure strategies given by $x=0$ and $x=1$ are also Nash equilibria and both are attracting, in which case one speaks of bistability.

For $n=3$, the classification of all phase portraits was achieved by Zeeman Ze80 (for the generic case) and by Bomze [Bo83], Bo94]. A basic result is that there exist no isolated periodic orbits and hence no limit cycles [Ho81. (In non-generic cases families of non-isolated periodic orbits can cover part or all of int $S_{3}$.) There are 33 generic phase portraits (or 19 up to flow reversal). Of particular interest is the case of the rock-scissors-paper game, where strategy 1 is dominated by 2 (in the absence of 3 , i.e., if $x_{3}=0$ ), and similarly 2 is dominated by 3 , and 3 is, in turn, dominated by 1 . After normalising by adding constants to the columns such that the diagonal terms are 0 , the payoff matrix is in this case of the form

$$
A=\left(\begin{array}{ccc}
0 & -a_{2} & b_{3} \\
b_{1} & 0 & -a_{3} \\
-a_{1} & b_{2} & 0
\end{array}\right)
$$


with $a_{i}$ and $b_{i}$ positive. There exists a unique rest point $\mathbf{z}$ in int $S_{3}$, which is also the unique Nash equilibrium of the corresponding game.

Theorem 2 ([Ze80]). The following conditions are equivalent for the rock-scissorspaper game given by (10):

(a) $\mathbf{z}$ is asymptotically stable,

(b) $\mathbf{z}$ is globally stable,

(c) $\operatorname{det} A>0$,

(d) $\mathbf{z}^{T} A \mathbf{z}>0$.

If $\operatorname{det} A=0$, then all orbits in $\operatorname{int} S_{n}$ are closed orbits around $\mathbf{z}$. If $\operatorname{det} A<0$, then all orbits in int $S_{n}$, apart from the rest point $\mathbf{z}$, converge to the boundary; see Figure 1. More precisely, for $\mathbf{x} \in \operatorname{int} S_{n}$, the $\omega$-limit (the set of accumulation points of $\mathbf{x}(t)$, for $t \rightarrow+\infty$ ) is the heteroclinic cycle consisting of the three saddle points $\mathbf{e}_{i}$ and the three edges which connect them (in the sense that these are orbits converging to one vertex for $t \rightarrow+\infty$ and to another for $t \rightarrow-\infty$ ). This is the simplest example showing that NE need not describe the outcome of the replicator dynamics.

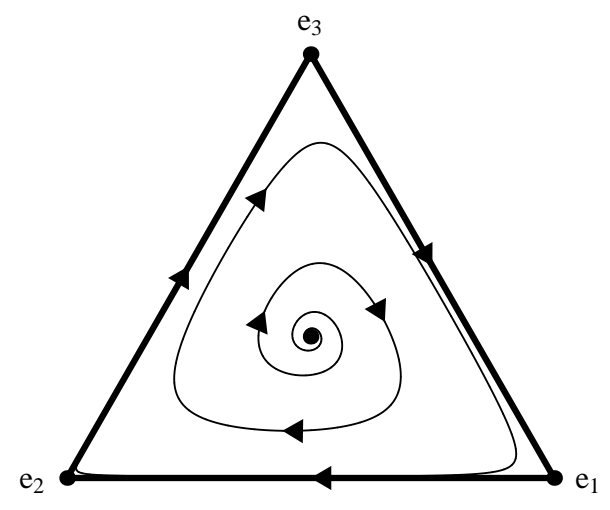

FiguRE 1. Replicator dynamics for the rock-scissors-paper game with payoff matrix (9) with $a_{i}=1$ and $b_{i}=0.55$.

For $n=4$, a complete classification seems out of reach. Examples show that there exist periodic attractors, and numerical simulations display chaotic attractors. The problem is equivalent to the classification of three-dimensional Lotka-Volterra equations. Indeed

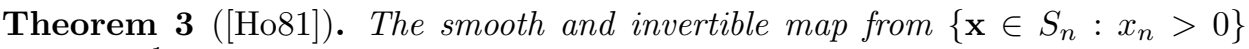
onto $\mathbf{R}_{+}^{n-1}$, given by $y_{i}=\frac{x_{i}}{x_{n}}$, maps the orbits of the replicator equation (3) onto the orbits of the Lotka-Volterra equation

$$
\dot{y}_{i}=y_{i}\left(r_{i}+\sum_{j} c_{i j} y_{j}\right)
$$

$i=1, \ldots, n-1$, where $r_{i}=a_{i n}-a_{n n}$ and $c_{i j}=a_{i j}-a_{n j}$.

The theorem allows us to use the large set of results on Lotka-Volterra equations, which are a basic model in mathematical ecology. On the other hand, an 
$n$-dimensional Lotka-Volterra equation (10) with equal basic growth rates $r_{i}=r$ reduces with $x_{i}=y_{i} /\left(y_{1}+\cdots+y_{n}\right)$ to the replicator equation (3) on $S_{n}$ with $c_{i j}=a_{i j}-\alpha$ (where $\alpha \in \mathbf{R}$ is arbitrary). In particular every replicator equation on $S_{n}$ can be imbedded into a competitive Lotka-Volterra equation on $\mathbf{R}_{+}^{n}$ (choose $r>0$ and $\alpha>0$ large enough so that $c_{i j}<0$ ), which has a globally attracting invariant manifold called the carrying simplex Hi88. In this sense the classification of [Z80] embeds into the classification of three-dimensional competitive LotkaVolterra equations of [Ze93]. However, the dynamics of (10) with different $r_{i}$ is in general richer than that with equal $r_{i}=r$, and the continua of periodic orbits can split up into several coexisting limit cycles; see [HSo94, Lu02]. For the present state of the art see [ZZ02, [ZZ03].

2.5 Permanence. The replicator equation is said to be permanent if there exists a compact set $K \subset \operatorname{int} S_{n}$ with the property that for all $\mathbf{x} \in \operatorname{int} S_{n}$ there is a $T$ such that for all $t>T$ one has $\mathbf{x}(t) \in K$. This means roughly that if initially all types (or strategies) are present in the population, then they will be, in the long run, proof against extinction through small, rare random shocks.

Theorem 4 (cf. [HoS88]). If (3) is permanent, then there exists a unique rest point $\mathbf{z} \in \operatorname{int} S_{n}$. The time averages along each interior orbit converge to $\mathbf{z}$ :

$$
\frac{1}{T} \int_{0}^{T} x_{i}(t) d t \longrightarrow z_{i}
$$

for $T \rightarrow+\infty$ and $i=1, \ldots, n$. If $a_{i i}=0$, then

$$
(-1)^{n-1} \operatorname{det} A>0, \quad \mathbf{z}^{T} A \mathbf{z}>0 .
$$

Conversely, if the replicator equation (3) has no rest point in int $S_{n}$, then every orbit converges to the boundary of $S_{n}$.

We note here that if an orbit in int $S_{n}$ has $\omega$-limit points on the boundary, its time-average need not converge. (For the rock-scissors-paper game, see section 3.5, and also [Gau92, [Ak93], GaH95]).

Theorem 5 (cf. HoS88]). The replicator equation (3) is permanent if there exists $a \mathbf{p} \in \operatorname{int} S_{n}$ such that

$$
\mathbf{p}^{T} A \mathbf{b}>\mathbf{b}^{T} A \mathbf{b}
$$

for all rest points $\mathbf{b} \in \mathrm{bd} S_{n}$.

Since it is actually enough to check the inequality for the extremal points of the rest points on the boundary (a union of convex sets), this yields a finite system of linear inequalities for $\mathbf{p}$.

Among the many examples of replicator equations we single out those given by the class of monocyclic payoff matrices $A$ (an $n$-strategy generalisation of the rockpaper-scissors case). Such matrices are defined by $a_{i i}=0, a_{i j}>0$ if $i=j+1$ $(\bmod n)$ and $a_{i j} \leq 0$ otherwise. For (3) this implies the existence of a heteroclinic cycle along the edges $1 \rightarrow 2 \rightarrow \cdots \rightarrow n \rightarrow 1$ which is attracting within bd $S_{n}$. The necessary and sufficient conditions for permanence contained in the previous two theorems coincide.

Theorem 6 ([HoS88]). The replicator equation with monocyclic $A$ is permanent iff there is a rest point $\mathbf{z} \in \operatorname{int} S_{n}$ with $\mathbf{z}^{T} A \mathbf{z}>0$. 
2.6 Mixed strategy dynamics and ESS. So far, we have implicitly assumed that the 'types' in the population correspond to the pure strategies given by the basis vectors $\mathbf{e}_{i}$ spanning the simplex $S_{n}$. Let us suppose now that the types may also correspond to mixed strategies $\mathbf{p}(i) \in S_{n}$, with $i=1, \ldots, N$ (we need not assume $N=n)$. The average payoff for an individual of type $\mathbf{p}(i)$ against an individual of type $\mathbf{p}(j)$ is $u_{i j}=\mathbf{p}(i)^{T} A \mathbf{p}(j)$, and if $\mathbf{x} \in S_{N}$ describes the types' frequencies in the population, then the average strategy within the population is $\mathbf{p}(\mathbf{x})=\sum x_{i} \mathbf{p}(i)$. The induced replicator equation $\dot{x}_{i}=x_{i}\left((U \mathbf{x})_{i}-\mathbf{x}^{T} U \mathbf{x}\right)$ can be written as

$$
\dot{x}_{i}=x_{i}\left[(\mathbf{p}(i)-\mathbf{p}(\mathbf{x}))^{T} A \mathbf{p}(\mathbf{x})\right] .
$$

The best-known concept of evolutionary game theory is that of evolutionary stability (see [MS74, [MS82, [Hi87] and the literature cited there). Intuitively speaking, if all members in the population use such an evolutionarily stable strategy, or ESS, then no 'mutant' minority using another strategy can invade. A strategy $\hat{\mathbf{p}} \in S_{n}$ is said to be evolutionarily stable if for every $\mathbf{p} \in S_{n}$ with $\mathbf{p} \neq \hat{\mathbf{p}}$, the induced replicator equation describing the dynamics of the population consisting of these two types only (the resident using $\hat{\mathbf{p}}$ and the invader using $\mathbf{p}$ ) leads to the elimination of the invader as long as the initial frequency of this invader is sufficiently small, i.e. below some 'invasion barrier' $\varepsilon(\mathbf{p})$. By (8) this equation reads (if $x$ is the frequency of the invader):

$$
\dot{x}=x(1-x)\left[x\left(\mathbf{p}^{T} A \mathbf{p}-\hat{\mathbf{p}}^{T} A \mathbf{p}\right)-(1-x)\left(\hat{\mathbf{p}}^{T} A \hat{\mathbf{p}}-\mathbf{p}^{T} A \hat{\mathbf{p}}\right)\right]
$$

and hence the rest point $x=0$ is asymptotically stable iff the following conditions are satisfied:

(a) (equilibrium condition)

$$
\mathbf{p}^{T} A \hat{\mathbf{p}} \leq \hat{\mathbf{p}}^{T} A \hat{\mathbf{p}}
$$

(b) (stability condition)

$$
\text { if } \mathbf{p}^{T} A \hat{\mathbf{p}}=\hat{\mathbf{p}}^{T} A \hat{\mathbf{p}}, \quad \text { then } \mathbf{p}^{T} A \mathbf{p}<\hat{\mathbf{p}}^{T} A \mathbf{p} .
$$

The first condition means that $\hat{\mathbf{p}}$ is a Nash equilibrium: no invader does better than the resident against the resident. The second condition states that if the invader does as well as the resident against the resident, then it does less well than the resident against the invader. (Note that $x=0$ may well be asymptotically stable, and hence $\hat{\mathbf{p}}$ is an ESS, if the replicator dynamics (15) is bistable: in this case, type $\mathbf{p}$ can invade if it enters the population with a frequency which is sufficiently high - viz., larger than the 'invasion barrier' $\varepsilon(\mathbf{p})$.)

Theorem 7 ([oSS79]). The strategy $\hat{\mathbf{p}}$ is an ESS iff $\prod_{i} x_{i}^{\hat{p}_{i}}$ is a strict local Lyapunov function for the replicator equation, or equivalently iff

$$
\hat{\mathbf{p}}^{T} A \mathbf{p}>\mathbf{p}^{T} A \mathbf{p}
$$

for all $\mathbf{p} \neq \hat{\mathbf{p}}$ in some neighborhood of $\hat{\mathbf{p}}$. If $\hat{\mathbf{p}} \in \operatorname{int} S_{n}$, then (18) holds for all $\mathbf{p} \in S_{n}$.

(The function $V(\mathbf{x})$ is said to be a Lyapunov function if $\dot{V}(\mathbf{x}) \geq 0$ for all $\mathbf{x}$, and strict if equality holds only when $\mathbf{x}$ is a rest point.)

In particular, an ESS is an asymptotically stable rest point, and an interior ESS is globally stable. The converse does not hold in general. But

Theorem 8 ([Cr90, Cr92]). The strategy $\hat{\mathbf{p}} \in S_{n}$ is an ESS iff it is strongly stable. 
Here, $\hat{\mathbf{p}}$ is said to be strongly stable if, whenever it is in the convex hull of $\mathbf{p}(1), \ldots, \mathbf{p}(N) \in S_{n}$, the strategy $\mathbf{p}(\mathbf{x}(t))$ converges to $\hat{\mathbf{p}}$, under (14), for all $\mathbf{x} \in S_{N}$ for which $\mathbf{p}(\mathbf{x})$ is sufficiently close to $\hat{\mathbf{p}}$.

The relation between evolutionary and dynamic stability is particularly simple for the class of partnership games, defined by $A=A^{T}$, for which the interests of both players coincide. For such games, $\hat{\mathbf{p}}$ is an ESS iff it is asymptotically stable for (3). This holds iff it is a strict local maximum of the average payoff $\mathbf{x}^{T} A \mathbf{x}$.

Many interesting games have no ESS. Often, it is useful to consider a generalisation (see [Th85], [BoP89], [Sw92], Bo98], BaS00, $\mathrm{Br} 03]$ ): a set $G \subset S_{n}$ is said to be an $E S$ set if for all $\hat{\mathbf{x}} \in G$ and all $\mathbf{x} \in S_{n}$

$$
\mathbf{x}^{T} A \hat{\mathbf{x}} \leq \hat{\mathbf{x}}^{T} A \hat{\mathbf{x}}
$$

holds, and if for all $\hat{\mathbf{x}} \in G$ and $\mathbf{x} \in S_{n} \backslash G$ for which equality holds,

$$
\mathbf{x}^{T} A \mathbf{x}<\hat{\mathbf{x}}^{T} A \mathbf{x}
$$

A singleton set $G=\{\hat{\mathbf{x}}\}$ is an ES set iff $\hat{\mathbf{x}}$ is an ESS. All elements of an ES set $G$ are NE which are neutrally stable in the sense that for $\mathbf{x}, \hat{\mathbf{x}} \in G$ the equality $\hat{\mathbf{x}}^{T} A \mathbf{x}=\mathbf{x}^{T} A \mathbf{x}$ holds whenever $\mathbf{x}^{T} A \hat{\mathbf{x}}=\hat{\mathbf{x}}^{T} A \hat{\mathbf{x}}$. A set $G$ is an ES set iff each $\hat{\mathbf{x}} \in G$ has a neighborhood $U$ such that $\mathbf{x}^{T} A \mathbf{x} \leq \hat{\mathbf{x}}^{T} A \mathbf{x}$ with equality iff $\mathbf{x} \in G$. If $G$ contains an $\hat{\mathbf{x}} \in \operatorname{int} S_{n}$, then $U$ can be chosen to be $S_{n}$. An ES set $G$ is uninvadable in the sense that there exists an $\epsilon>0$ such that any strategy $\hat{\mathbf{x}} \in G$ cannot be invaded by a minority of $\mathbf{x} \notin G$ as long as its frequency is below $\epsilon$.

Any strategy in an ES set is stable, and any ES set is asymptotically stable. If an ES set $G$ contains a point $\hat{\mathbf{x}}$ in int $S_{n}$, then all orbits in the interior of $S_{n}$ converge to $G$ (see Cr03]).

2.7 Bimatrix games. Let us return now to asymmetric games defined by two matrices $A$ and $B$, also called bimatrix games. If the two roles correspond to two populations, the ansatz leading to the replicator equation now yields

$$
\begin{gathered}
\dot{x}_{i}=x_{i}\left[(A \mathbf{y})_{i}-\mathbf{x}^{T} A \mathbf{y}\right] \\
\dot{y}_{j}=y_{j}\left[\left(B^{T} \mathbf{x}\right)_{j}-\mathbf{x}^{T} B \mathbf{y}\right]
\end{gathered}
$$

on $S_{n} \times S_{m}$. Again, faces and interiors are invariant. In [EsAk83] and [HoS88], HoSi98 it is shown that up to a change in velocity, the corresponding flow in $\operatorname{int}\left(S_{n} \times S_{m}\right)$ is incompressible. Hence there can be no attractors (and in particular no asymptotically stable rest point) in the interior. Indeed, a rest point is asymptotically stable iff it is a strict NE. (These results extend from two-player games to $N$-player games [RiW95], but others go wrong for $N \geq 3$ due to nonlinearity of the payoff functions; see [P197]). A good way to analyze (21)-(22) is to transform it into a bipartite system of the form

$$
\dot{u}=f(v), \quad \dot{v}=g(u), \quad u \in \mathbf{R}^{n-1}, v \in \mathbf{R}^{m-1} .
$$

From this representation the preservation of volume and the Hamiltonian character of the linearization near interior equilibria become obvious Ho96.

Again, in two dimensions, i.e. for $n=m=2$, a complete classification of phase portraits of (21)-(22) is available, see [HoS88], [HoSi98] for the four generic cases and $\mathrm{Cr} 03$ for the degenerate cases.

In analogy to ES sets, $S E$ (strict equilibria) sets are defined as sets $G \subseteq S_{n} \times S_{m}$ of Nash equilibria such that $\hat{\mathbf{x}}^{T} A \hat{\mathbf{y}}>\mathbf{x}^{T} A \hat{\mathbf{y}}$ whenever $(\hat{\mathbf{x}}, \hat{\mathbf{y}}) \in G$ and $(\mathbf{x}, \hat{\mathbf{y}}) \notin G$, and similarly with $\mathbf{x}$ and $\mathbf{y}$ interchanged (see [Cr03] . This is equivalent to defining 
an SE set $G$ as a set of Nash equilibria such that whenever $\mathbf{x}^{T} A \hat{\mathbf{y}}=\hat{\mathbf{x}}^{T} A \hat{\mathbf{y}}$, then $(\mathbf{x}, \hat{\mathbf{y}}) \in G$ and similarly with $\mathbf{x}$ and $\mathbf{y}$ interchanged. Strict NE are exactly the singleton SE sets, and generalising the singleton situation, a set of rest points is asymptotically stable iff it is an SE set. Such sets are finite unions of products of faces of $S_{n}$ and $S_{m}$. If $A=B$ and $G$ is an SE set, then the first component of its intersection with the diagonal of $S_{n} \times S_{n}$ is either empty or an ES set Cr03.

The canonical way to turn a bimatrix game $(A, B)$ into a symmetric game consists in assuming that chance decides which player is in which role: role I will be adopted with probability $p$ (with $0<p<1$ ). The players' strategies must therefore allow for both situations and are of the form $(i, j)$ : in role I, play $i \in \operatorname{Strat}(I)$, in role II play $j \in \operatorname{Strat}(I I)$. The payoff matrix $C$ is an $n m \times n m$ matrix, with $c_{i j, k l}:=p a_{i l}+(1-p) b_{k j}$. The symmetric game with this matrix is said to be the symmetrized version of the bimatrix game. For any $\mathbf{z}=\left(z_{i j}\right) \in S_{n m}$, marginals $\mathbf{x} \in S_{n}$ and $\mathbf{y} \in S_{m}$ are defined by $x_{i}:=\sum_{j} z_{i j}$ and $y_{j}:=\sum_{i} z_{i j}$. Conversely, for any given $\mathbf{x} \in S_{n}$ and $\mathbf{y} \in S_{m}$, there is at least one $\mathbf{z} \in S_{n m}$ such that $\mathbf{x}$ and $\mathbf{y}$ are its marginals, namely $z_{i j}:=x_{i} y_{j}$.

There exists a symmetric NE $\hat{\mathbf{z}} \in S_{n m}$ for the symmetrized game with matrix $C$. Since $\hat{\mathbf{z}}$ is a best reply to itself,

$$
\mathbf{z}^{T} C \hat{\mathbf{z}} \leq \hat{\mathbf{z}}^{T} C \hat{\mathbf{z}}
$$

for all $\mathbf{z} \in S_{n m}$. Hence

$$
p \mathbf{x}^{T} A \hat{\mathbf{y}}+(1-p) \hat{\mathbf{x}}^{T} B \mathbf{y} \leq p \hat{\mathbf{x}}^{T} A \hat{\mathbf{y}}+(1-p) \hat{\mathbf{x}}^{T} B \hat{\mathbf{y}}
$$

In particular, if $\mathbf{x}=\hat{\mathbf{x}}$ and $\mathbf{y}$ is arbitrary, this implies that $\hat{\mathbf{y}}$ is a best reply to $\hat{\mathbf{x}}$ and vice versa; i.e. $(\hat{\mathbf{x}}, \hat{\mathbf{y}})$ is an NE.

The replicator equation on $S_{n m}$ is

$$
\dot{z}_{i j}=z_{i j}\left[\left(\mathbf{e}_{i}, \mathbf{f}_{j}\right)-\mathbf{z}\right]^{T} C \mathbf{z} .
$$

Since $\left(z_{i j} / z_{i l}\right)=(1-p)\left(z_{i j} / z_{i l}\right) \mathbf{x}^{T} B\left(\mathbf{f}_{j}-\mathbf{f}_{l}\right)$ the quotient $\frac{z_{i j} z_{k l}}{z_{i l} z_{k j}}$ is a constant of motion. Thus $S_{n m}$ foliates into invariant submanifolds (see GaHS91, [CrGW00, Cr03]). In particular, since the set $\left\{\mathbf{z} \in S_{n m}: z_{i j} z_{k l}=z_{i l} z_{k j}, 1 \leq i, k \leq n\right.$, $1 \leq j, l \leq m\}$ is just the so-called Wright manifold $W=\left\{\mathbf{z} \in S_{n m}: z_{i j}=x_{i} y_{j}\right.$, $1 \leq i \leq n, 1 \leq j \leq m\}$, this $(n+m-2)$-dimensional submanifold of $S_{n m}$ is invariant. On this set, the average strategies in the two roles are independent. The dynamics (26) simplifies on $W$ to yield

$$
\dot{x}_{i}=p x_{i}\left[(A \mathbf{y})_{i}-\mathbf{x}^{T} A \mathbf{y}\right]
$$

and

$$
\dot{y}_{j}=(1-p) y_{j}\left[\left(B^{T} \mathbf{x}\right)_{j}-\mathbf{x}^{T} B \mathbf{y}\right]
$$

on $S_{n} \times S_{m}$. Up to the positive factors $p$ and $1-p$, this is just the two-population replicator equation (21)-(22).

In the case $n=m=2,(26)$ is a replicator equation whose matrix, after adding appropriate constants to the columns, is of the form

$$
M=\left(\begin{array}{cccc}
0 & 0 & 0 & 0 \\
R & R & S & S \\
R+r & R+s & S+s & S+r \\
r & s & s & r
\end{array}\right)
$$


The signs of $R, S, r$ and $s$ yield the orientation of the flow on the edges $\mathbf{e}_{1} \mathbf{f}_{1}-$ $\mathbf{e}_{2} \mathbf{f}_{1}-\mathbf{e}_{2} \mathbf{f}_{2}-\mathbf{e}_{1} \mathbf{f}_{2}-\mathbf{e}_{1} \mathbf{f}_{1}$ spanning the invariant manifolds $W_{K}=\left\{\mathbf{z} \in S_{4}: z_{11} z_{22}=\right.$ $\left.K z_{21} z_{12}\right\}$ (for each $K>0$ ) and determine the phase portraits GaHS91. Rest points in the interior of $S_{4}$ (one on each $W_{K}$ ) exist iff $R S>0$ and $r s>0$. If $R r>0$, the dynamics is bistable: all interior rest points are of saddle type (within their manifold $W_{K}$ ), and up to a set of measure zero, all orbits converge to one of two opposite corner points of $S_{4}$. If $R r<0$, we obtain the cyclic $2 \times 2$-game where the flow-induced orientations of the edges form a cycle: $W_{1}$ is filled in this case with periodic orbits surrounding the rest point, whereas generically, if $K>0$ is on one side of 1 , all orbits on $W_{K}$ spiral towards the interior rest point, and if $K$ is on the other side, all orbits spiral away from it and towards the heteroclinic cycle formed by the edges spanning $W_{K}$.

In general, a set $G \subseteq S_{n m}$ is an ES set of the symmetrized game iff $G=\{\mathbf{z}$ : $(\mathbf{x}, \mathbf{y}) \in H\}$ where $\mathbf{x} \in S_{n}$ and $\mathbf{y} \in S_{m}$ are the marginals of $\mathbf{z}$ and $H$ is an SE set of the bimatrix game. This implies that each ESS of the symmetrized game corresponds to a strict NE pair $\left(\mathbf{e}_{i}, \mathbf{f}_{j}\right)$ of the asymmetric game. The ES sets are exactly the asymptotically stable sets of rest points of the symmetrized replicator equation (26). A mixed NE of the symmetrized game cannot be an ESS Se80.

A bimatrix game $(A, B)$ is said to be a c-partnership game, resp. c-zerosum game (for some $c>0$ resp. $c<0$ ), if there exist suitable constants $d_{i j}, c_{j}$ and $d_{i}$ such that

$$
a_{i j}=d_{i j}+c_{j} \quad \text { and } \quad b_{i j}=c d_{i j}+d_{i} .
$$

Such games have the same Nash equilibria as the games $(D, D)$, resp. $(D,-D)$. If there exists an NE pair $(\hat{\mathbf{x}}, \hat{\mathbf{y}})$ in the interior of $S_{n} \times S_{m}$, then the function

$$
H(\mathbf{x}, \mathbf{y})=c \sum \hat{x}_{i} \log x_{i}-\sum \hat{y}_{j} \log y_{j}
$$

is a constant of motion for (21)-(22) and even a Hamiltonian function. In particular, an interior equilibrium of a $c$-zerosum game is always stable (but not asymptotically stable).

Theorem 9 ([HoS88], MoSh96a], HoSi98]). The game $(A, B)$ is a c-partnership game iff one of the following conditions holds:

(i) for all $i, k \in\{1, \ldots, n\}$ and $j, l \in\{1, \ldots, m\}$

$$
c\left(a_{i j}-a_{i l}-a_{k j}+a_{k l}\right)=b_{i j}-b_{i l}-b_{k j}+b_{k l} ;
$$

(ii) there exist $u_{i}, v_{j}$ such that $Q=c A-B$ satisfies $q_{i j}=u_{i}+v_{j}$ for all $i$ and $j$;

(iii) for all $\boldsymbol{\xi} \in \mathbf{R}_{0}^{n}$ and all $\boldsymbol{\eta} \in \mathbf{R}_{0}^{m}$

$$
c \boldsymbol{\xi}^{T} A \boldsymbol{\eta}=\boldsymbol{\xi}^{T} B \boldsymbol{\eta} .
$$

A related result holds for $N$-person games; see [MoSh96a].

For games with two populations, the usual interpretation of evolutionary stability makes little sense, since invaders from one population do not interact with their own population. A weak analog is the following. A pair of strategies $(\hat{\mathbf{x}}, \hat{\mathbf{y}})$ is said to be a Nash-Pareto pair if it is a Nash equilibrium and if, in addition, for all states $(\mathbf{x}, \mathbf{y}) \in S_{n} \times S_{m}$ with $\mathbf{x} \in B R(\hat{\mathbf{y}})$ and $\mathbf{y} \in B R(\hat{\mathbf{x}})$,

$$
\text { if } \mathbf{x}^{T} A \mathbf{y}>\hat{\mathbf{x}}^{T} A \mathbf{y}, \quad \text { then } \quad \mathbf{x}^{T} B \mathbf{y}<\mathbf{x}^{T} B \hat{\mathbf{y}}
$$

$$
\text { if } \quad \mathbf{x}^{T} B \mathbf{y}>\mathbf{x}^{T} B \hat{\mathbf{y}}, \quad \text { then } \quad \mathbf{x}^{T} A \mathbf{y}<\hat{\mathbf{x}}^{T} A \mathbf{y} .
$$


Thus it is impossible that both players get an advantage by deviating from the equilibrium.

Theorem 10 ([HoS88] $).(\hat{\mathbf{x}}, \hat{\mathbf{y}}) \in \operatorname{int}\left(S_{n} \times S_{m}\right)$ is a Nash-Pareto pair iff there exists a constant $c>0$ such that

$$
c(\mathbf{x}-\hat{\mathbf{x}})^{T} A \mathbf{y}+\mathbf{x}^{T} B(\mathbf{y}-\hat{\mathbf{y}})=0
$$

for all $(\mathbf{x}, \mathbf{y}) \in \operatorname{int}\left(S_{n} \times S_{m}\right)$, i.e. iff $(A, B)$ is a (-c)-zerosum game. Such a NashPareto-pair is stable for the replicator equation (21)-(22).

In this case, (21)-(22) is a Hamiltonian system with respect to a suitable Poisson structure. The dynamics on the energy levels can be complicated; see Sat02 for chaos in an asymmetric rock-scissors-paper game. For general bimatrix games, a normal form analysis near interior equilibria for $n=m=3$ shows their generic instability [Ho96]. This suggests the

Conjecture. If an interior equilibrium is isolated and stable under (21)-(22), then it is a Nash-Pareto pair.

\section{OTHER GAME DYNAMICS}

3.1 Nonlinear payoff functions. We have assumed so far that the average payoff to strategy $i$ is given by a linear function $(A \mathbf{x})_{i}$. This makes sense if the interactions are pairwise, with co-players chosen randomly within the population. But many interesting examples lead to non-linear payoff functions $a_{i}(\mathbf{x})$, for instance if the interactions occur in groups with more than two members. This leads to the replicator equation

$$
\dot{x}_{i}=x_{i}\left(a_{i}(\mathbf{x})-\bar{a}\right)
$$

on $S_{n}$, where $\bar{a}=\sum_{i} x_{i} a_{i}(\mathbf{x})$ is again the average payoff within the population. Many of the previous results can be extended in a straightforward way, sometimes in a localised version. For instance, the dynamics is unchanged under addition of a function $\psi$ to all payoff functions $a_{i}$. The existence of Nash equilibria can be shown as in Theorem 1 by perturbing (37) (see $\mathrm{HoSi98}_{1}$ ), and a straight extension of the folk theorem is still valid. An $\hat{\mathbf{x}}$ is said to be a local ESS if $\hat{\mathbf{x}}^{T} \mathbf{a}(\mathbf{x})>\mathbf{x}^{T} \mathbf{a}(\mathbf{x})$ for all $\mathbf{x} \neq \hat{\mathbf{x}}$ in some neighborhood of $\hat{\mathbf{x}}$ [Th85]. It can be characterised by a localised version of the equilibrium and stability condition, and it is an asymptotically stable rest point of (37). There are several look-alike contenders for the notion of an ES set (see [Th85, [Bo98] and [BaS00]).

An important class of payoff functions is given by potentials. For this, it is useful to introduce a Riemannian metric (the so-called Shahshahani metric) in the interior of $S_{n}$ by the inner product

$$
(\boldsymbol{\xi}, \boldsymbol{\eta})_{\mathbf{x}}=\sum \frac{1}{x_{i}} \xi_{i} \eta_{i}
$$

for the vectors $\boldsymbol{\xi}$ and $\boldsymbol{\eta}$ belonging to $\mathbf{R}_{0}^{n}=\left\{\boldsymbol{\xi} \in \mathbf{R}^{n}: \sum \xi_{i}=0\right\}$, i.e. to the tangent space of $\mathbf{x} \in \operatorname{int} S_{n}$ (see [Ak79]). Equation (37) is a Shahshahani gradient if there exists a potential function $V$, in the sense that

$$
(\dot{\mathbf{x}}, \boldsymbol{\xi})_{\mathbf{x}}=D_{\mathbf{x}} V(\boldsymbol{\xi})
$$


for all $\boldsymbol{\xi} \in \mathbf{R}_{0}^{n}$. In [HoS88] it is shown that this is the case iff

$$
\frac{\partial a_{i}}{\partial x_{j}}+\frac{\partial a_{j}}{\partial x_{k}}+\frac{\partial a_{k}}{\partial x_{i}}=\frac{\partial a_{i}}{\partial x_{k}}+\frac{\partial a_{k}}{\partial x_{j}}+\frac{\partial a_{j}}{\partial x_{i}}
$$

for all $i, j, k \in\{1, \ldots, n\}$, a condition which is trivially satisfied if $n=2$. If the payoff matrix $A$ describes a partnership game (i.e. $A=A^{T}$ ), then $V(\mathbf{x})=\frac{1}{2} \mathbf{x}^{T} A \mathbf{x}$ is such a potential, and the induced equation (14) for the mixed types is also a Shahshahani gradient [Si87]. For interesting applications to optimization problems see [Bo02. For bimatrix games, an obvious variant can be introduced; the replicator equation (21)-(22) is then a gradient for the $c$-partnership games, with potential function $\mathbf{x}^{T} D \mathbf{y}$ with $D$ given by (30).

As with bimatrix games, non-linear two-population games can be symmetrized, the dynamics admits invariant submanifolds, etc. Of particular interest for ecological scenarios are payoff functions which depend, not only on the frequency of the strategies in the other population, but also on the strategy distribution in the resident population, and on the densities of one or both populations. For this we refer to [Cr95], Cr03], and for the $N$-species case to [CrGH01].

3.2 Imitation dynamics. Strategies can be transmitted within a population through imitation. Such a process can be modelled in many ways. Following Weibull [We95], let us first assume that individuals occasionally chose at random another player in the population, and adopt the strategy of this 'model' with a certain probability which can depend on the payoff difference, the frequency of the strategies, etc. This ansatz yields an input-output model

$$
\dot{x}_{i}=x_{i} \sum_{j}\left[f_{i j}(\mathbf{x})-f_{j i}(\mathbf{x})\right] x_{j}
$$

with $f_{i j}$ as the rate at which a player of type $j$ adopts type $i$; see Ho95b, HoSi98. A plausible assumption is that this rate depends only on the payoffs achieved by the two players, i.e.

$$
f_{i j}(\mathbf{x})=f\left(a_{i}(\mathbf{x}), a_{j}(\mathbf{x})\right)
$$

where $f(u, v)$ defines the imitation rule (the same for all players). The simplest rule is to imitate the better, i.e.

$$
f(u, v)=0 \quad \text { if } \quad u<v \quad \text { and } \quad f(u, v)=1 \quad \text { if } \quad u>v,
$$

which however leads to a discontinuous right hand side. In this case a strategy increases iff its payoff is larger than the median of the payoff values $a_{1}(\mathbf{x}), \ldots, a_{n}(\mathbf{x})$ [FL98 (whereas it increases for the replicator equation iff it exceeds the mean). In a region of $S_{n}$ defined by a given rank-ordering of the payoff values (for instance $\left.a_{1}(\mathbf{x})>a_{2}(\mathbf{x})>\cdots>a_{n}(\mathbf{x})\right)$, the dynamics reduces to a replicator equation with a skew-symmetric matrix $A$ consisting only of 0 's and \pm 1 's (in the example, $a_{i j}=1$ if $j>i, a_{i j}=-1$ for $j<i$, and $a_{i i}=0$ ); see Ho95b. Figure 2 describes the phase portrait of a rock-scissors-paper game for this dynamic.

The assumption in (42) that $f(u, v)$ is an increasing function $\phi(u-v)$ of the payoff difference is also plausible. This leads to imitation dynamics of the form

$$
\dot{x}_{i}=x_{i} \sum_{j} \psi\left(a_{i}(\mathbf{x})-a_{j}(\mathbf{x})\right) x_{j}
$$




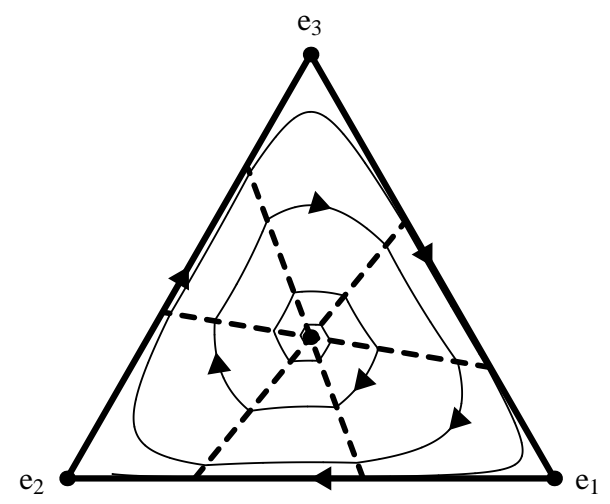

FiguRE 2. Imitate the better dynamics for the rock-scissors-paper game with payoff matrix (9) with $a_{i}=1$ and $b_{i}=0.55$.

with an increasing and odd function $\psi$. In particular, choosing $\phi(z)=0$ for $z \leq 0$ and $\phi(z)=\alpha z$ for $z>0$ (and some positive constant $\alpha$ ) turns (44) into the replicator equation (37). If players use this rule (the proportional imitation rule of [Sc97; see also [Cr03]), they imitate strategies with a higher payoff, with a probability which is proportional to the expected gain obtained by switching. A more general approach leads to

$$
\dot{x}_{i}=x_{i}\left[f\left(a_{i}(\mathbf{x})\right)-\sum x_{j} f\left(a_{j}(\mathbf{x})\right)\right]
$$

for some strictly increasing function $f$. This equation arises for the imitation rule $f(u, v)=f(u)-f(v)$. If $f$ is linear, one obtains again the replicator equation (37). Similarly, for the imitation rules $f(u, v)=f(u)-c$, or for $f(u, v)=c-f(v)$, i.e. if the rate depends only on the payoff of the imitating or of the imitated player, one obtains the equation (45).

The most general form of an imitation dynamics is given by

$$
\dot{x}_{i}=x_{i} g_{i}(\mathbf{x})
$$

where the functions $g_{i}$ satisfy $\sum x_{i} g_{i}(\mathbf{x})=0$ on $S_{n}$. The simplex $S_{n}$ and its faces are invariant. Such an equation is said to be payoff monotonic [Fr91], We95] if

$$
g_{i}(\mathbf{x})>g_{j}(\mathbf{x}) \Leftrightarrow a_{i}(\mathbf{x})>a_{j}(\mathbf{x}) .
$$

All imitation dynamics encountered so far have this property. For payoff monotonic equations (46) the folk theorem holds again: NE are rest points, strict NE are asymptotically stable, and rest points that are stable or $\omega$-limits of interior orbits are NE.

The dynamics (46) is said to be aggregate monotonic [SaZ92] if

$$
\mathbf{y}^{T} \mathbf{g}(\mathbf{x})>\mathbf{z}^{T} \mathbf{g}(\mathbf{x}) \Longleftrightarrow \mathbf{y}^{T} \mathbf{a}(\mathbf{x})>\mathbf{z}^{T} \mathbf{a}(\mathbf{x})
$$

for all $\mathbf{x}, \mathbf{y}, \mathbf{z} \in S_{n}$. It turns out that all aggregate monotonic imitation dynamics reduce (through a change in velocity) to replicator dynamics (37). Cressman [Cr97] shows that the linearization at a rest point of a payoff monotonic dynamic (47) is proportional to that of the replicator dynamics. In particular, regular ESS are 
asymptotically stable for any smooth dynamics (47) satisfying some mild regularity condition.

A pure strategy $i$ is said to be strictly dominated if there exists some $\mathbf{y} \in S_{n}$ such that

$$
a_{i}(\mathbf{x})<\mathbf{y}^{T} \mathbf{a}(\mathbf{x})
$$

for all $\mathbf{x} \in S_{n}$. A rational player will not use such a strategy. If such strategies are eliminated, it may happen that in the reduced game, some additional strategies are strictly dominated. One may repeat this elimination procedure a finite number of times. The strategies eliminated this way are said to be iteratively strictly dominated. If all players are rational and this is common knowledge among them, these strategies will be not be used.

For a large class of evolutionary dynamics, iteratively strictly dominated strategies can similarly be discarded, even if players are not assumed to be rational. More precisely, this holds if game dynamics (46) is convex monotone in the sense that

$$
a_{i}(\mathbf{x})<\mathbf{y}^{T} \mathbf{a}(\mathbf{x}) \Rightarrow g_{i}(\mathbf{x})<\mathbf{y}^{T} \mathbf{g}(\mathbf{x})
$$

for all $i$ and all $\mathbf{x}, \mathbf{y} \in S_{n}$.

Theorem 11 ([HoW96]). If the game dynamics (46) is convex monotone and strategy $i$ is iteratively strictly dominated, then $x_{i}(t) \rightarrow 0$ for $t \rightarrow+\infty$ along interior solutions.

If the dominating strategy $\mathbf{y}$ in (49) is pure, then this result follows already from (47). Thus selection eliminates strictly dominated strategies just as rational players would do. However, this appealing property holds for fewer dynamics than one might expect. An equation of type (45) is convex monotone iff $f$ is convex. If $f$ is not convex, there exist games with strictly dominated strategies that survive along an open set of orbits; see HoW96, HoSi98. For the other class of imitation dynamics (44) the situation is even worse: For essentially all nonlinear $\psi$ survival of strictly dominated strategies is possible (see $[\mathrm{Ho95b}$ and for related results $[\mathrm{Se} 98]$ ).

HoSc00 studies imitation dynamics where imitators observe not one but $N$ individuals. For cyclic $2 \times 2$ games this stabilizes the equilibrium for $N \geq 2$, and in the limit $N \rightarrow \infty$ this yields

$$
\begin{aligned}
\dot{x}_{i} & =x_{i}\left[a_{i}(\mathbf{y}) / \mathbf{x}^{T} \mathbf{a}(\mathbf{y})-1\right] \\
\dot{y}_{j} & =y_{j}\left[b_{j}(\mathbf{x}) / \mathbf{y}^{T} \mathbf{b}(\mathbf{x})-1\right],
\end{aligned}
$$

which is Maynard Smith's version of the two-population replicator equation [MS82].

We conclude with some open problems from [Ho95b]: Are interior ESS globally stable for (45) with convex $f$ ? This holds for $n=3$. For every nonconvex $f$ there are counterexamples. Does (44) have a constant of motion for zero-sum games? Again, this holds for $n=3$, even in the limit case of the 'imitate the better' rule (43).

3.3. Best response dynamics. Learning through imitation makes only modest requirements on the cognitive capabilities of the players. The best response dynamics GiM91, Ma92], Ho95a assumes more sophistication: in a large population, a small fraction of the players revise their strategy, choosing best replies $B R(\mathbf{x})$ to the current mean population strategy $\mathbf{x}$. This approach, which postulates that 
players are intelligent enough to gauge the current population state and to respond optimally, yields the best response (BR) dynamics

$$
\dot{\mathbf{x}} \in B R(\mathbf{x})-\mathbf{x} .
$$

Since best replies are in general not unique, this is a differential inclusion rather than a differential equation $\mathrm{AuC84}$. For continuous payoff functions $a_{i}(\mathbf{x})$ the right hand side is a non-empty, convex, compact subset of $S_{n}$ which is upper semicontinuous in $\mathbf{x}$. Hence solutions exist that are Lipschitz functions $\mathbf{x}(t)$ satisfying (51) for almost all $t \geq 0$.

If $B R(\mathbf{x})$ is a uniquely defined (and hence pure) strategy $\mathbf{b}$, the solution of (51) is given by

$$
\mathbf{x}(t)=\left(1-e^{-t}\right) \mathbf{b}+e^{-t} \mathbf{x}
$$

for small $t \geq 0$, which describes a linear orbit pointing straight towards the best reply. This can lead to a state where $\mathbf{b}$ is no longer the unique best reply. But for each $\mathbf{x}$ there always exists a $\mathbf{b} \in B R(\mathbf{x})$ which, among all best replies to $\mathbf{x}$, is a best reply against itself (i.e. an NE of the game restricted to the simplex $B R(\mathbf{x})$ ), and then $\mathbf{b} \in B R((1-\epsilon) \mathbf{x}+\epsilon \mathbf{b})$ holds for small $\epsilon \geq 0$ if the game is linear. An iteration of this construction yields at least one piecewise linear solution of (51) through $\mathbf{x}$ defined for all $t>0$. One can show [Ho95a] that for generic linear games essentially all solutions can be constructed in this way. For the resulting (multivalued) semi-dynamical system, the simplex $S_{n}$ is only forward invariant and bd $S_{n}$ need no longer be invariant: the frequency of strategies which are initially missing can grow, in contrast to the imitation dynamics. In this sense, the best response dynamics is an innovative dynamics.

For $n=2$, the phase portraits of (51) differ only in details from that of the replicator dynamics. If $\mathbf{e}_{1}$ is dominated by $\mathbf{e}_{2}$, there are only two orbits: the rest point $\mathbf{e}_{2}$, and the semi-orbit through $\mathbf{e}_{1}$ which converges to $\mathbf{e}_{2}$. In the bistable situation with interior NE $\mathbf{p}$, there are infinitely many solutions starting at $\mathbf{p}$ besides the constant one, staying there for some time and then converging monotonically to either $\mathbf{e}_{1}$ or $\mathbf{e}_{2}$. In the case of stable coexistence with interior NE $\mathbf{p}$, the solution starting at some point $\mathbf{x}$ between $\mathbf{p}$ and $\mathbf{e}_{1}$ converges toward $\mathbf{e}_{2}$ until it hits $\mathbf{p}$ and then remains there forever. (In the trivial game, with a continuum of equilibria, every Lipschitz curve in $S_{2}$ is a solution.)

For $n=3$, the differences to the replicator dynamics become more pronounced. In particular, for the rock-scissors-paper game given by (9), all orbits converge to the Nash equilibrium $\mathbf{p}$ whenever $\operatorname{det} A>0$ (just as with the replicator dynamics), but for $\operatorname{det} A<0$, all orbits (except possibly $\mathbf{p}$ ) converge to a limit cycle, the socalled Shapley triangle spanned by the three points $A_{i}$ (where $A_{1}$ is the solution of $(A \mathbf{x})_{2}=(A \mathbf{x})_{3}=0$, etc.); see Figure 3. In fact, the piecewise linear function $V(\mathbf{x}):=\left|\max _{i}(A \mathbf{x})_{i}\right|$ is a Lyapunov function for (51). In this case, the orbits of the replicator equation (3) converge to the boundary of $S_{n}$, but interestingly, the time averages

$$
\mathbf{z}(T):=\frac{1}{T} \int_{0}^{T} \mathbf{x}(t) d t
$$

have the Shapley triangle as a set of accumulation points, for $T \rightarrow+\infty$. Similar parallels between the best response dynamics and the behaviour of time-averages of the replicator equation are quite frequent; see [GaH95]. 


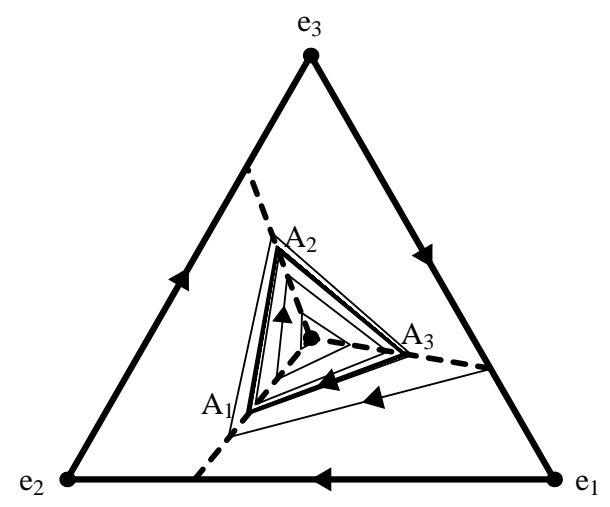

FiguRE 3. Best response dynamics for the rock-scissors-paper game with payoff matrix (9) with $a_{i}=1$ and $b_{i}=0.55$.

Obviously, strict NE are asymptotically stable, and strictly dominated strategies are eliminated along all solutions of the best response dynamics. For interior NE of linear games the following stability result is shown in Ho95a].

Let $\mathcal{B}=\left\{\mathbf{b} \in \operatorname{bd} S_{n}:(A \mathbf{b})_{i}=(A \mathbf{b})_{j}\right.$ for all $\left.i, j \in \operatorname{supp}(\mathbf{b})\right\}$ denote the set of all rest points of (3) on the boundary. Then the function

$$
w(\mathbf{x})=\max \left\{\sum_{\mathbf{b} \in \mathcal{B}} \mathbf{b}^{T} A \mathbf{b} u(\mathbf{b}): u(\mathbf{b}) \geq 0, \sum_{\mathbf{b} \in \mathcal{B}} u(\mathbf{b})=1, \sum_{\mathbf{b} \in \mathcal{B}} u(\mathbf{b}) \mathbf{b}=\mathbf{x}\right\}
$$

can be interpreted in the following way. Imagine the population in state $\mathbf{x}$ being decomposed into subpopulations of size $u(\mathbf{b})$ which are in states $\mathbf{b} \in \mathcal{B}$, and call this a $\mathcal{B}$-segregation of $\mathbf{b}$. Then $w(\mathbf{x})$ is the maximum mean payoff population $\mathbf{x}$ can obtain by such a $\mathcal{B}$-segregation. It is the smallest concave function satisfying $w(\mathbf{b}) \geq \mathbf{b}^{T} A \mathbf{b}$ for all $\mathbf{b} \in \mathcal{B}$.

Theorem 12 (

a. There is a vector $\mathbf{p} \in S_{n}$, such that $\mathbf{p}^{T} A \mathbf{b}>\mathbf{b}^{T} A \mathbf{b}$ holds for all $\mathbf{b} \in \mathcal{B}$.

b. $V(\mathbf{x})=\max _{i}(A \mathbf{x})_{i}-w(\mathbf{x})>0$ for all $\mathbf{x} \in S_{n}$.

c. There exist a unique interior equilibrium $\hat{\mathbf{x}}$ and $\hat{\mathbf{x}}^{T} A \hat{\mathbf{x}}>w(\hat{\mathbf{x}})$.

These conditions imply: $\hat{\mathbf{x}}$ is reached in finite and bounded time by any BR path.

The proof consists in showing that the function $V$ from (b) decreases along the paths of (51). Note that condition (a) is a sufficient condition for permanence of the replicator dynamics (3); see section 2.5. It is an open problem whether for generic payoff matrices $A$, permanence of the replicator equation is equivalent to the global stability of the interior equilibrium under the best response dynamics.

Let us discuss some examples of this general stability result. If $\mathbf{p}>\mathbf{0}$ is an interior ESS, then condition (a) holds not only for all $\mathbf{b} \in \mathcal{B}$ but for all $\mathbf{b} \neq \mathbf{p}$. In this case the simpler Lyapunov function $V(\mathbf{x})=\max _{i}(A \mathbf{x})_{i}-\mathbf{x}^{T} A \mathbf{x} \geq 0$ can also be used; see [Ho00]. With similar arguments, asymptotic stability of any boundary ESS can be shown.

In the rock-scissors-paper game, the set $\mathcal{B}$ reduces to the set of pure strategies, and the Lyapunov function is simply $V(\mathbf{x})=\max _{i}(A \mathbf{x})_{i}$. The same applies 
to the more general class of monocyclic matrices considered in section 2.5. For these games, the above conditions essentially characterize stability of the interior equilibrium.

All ES sets are forward invariant under the best response dynamics, but whether they are asymptotically stable is an open question; see [Cr03. Cressman [Cr03] computes the global attractor of the BR dynamics for certain extensive form games.

For bimatrix games the best response dynamics reads

$$
\dot{\mathbf{x}} \in B R(\mathbf{y})-\mathbf{x} \quad \dot{\mathbf{y}} \in B R(\mathbf{x})-\mathbf{y} .
$$

Modulo a time change it is equivalent to the continuous time version of fictitious play [Br51], in which Brown sketched a proof of convergence to equilibria for zerosum games using the Lyapunov function $V(\mathbf{x}, \mathbf{y})=\max _{i}(A \mathbf{y})_{i}-\min _{j}\left(\mathbf{x}^{T} A\right)_{j}$. Since

$$
\max _{i}(A \mathbf{y})_{i} \geq \mathbf{x}^{T} A \mathbf{y} \geq \min _{j}\left(\mathbf{x}^{T} A\right)_{j}
$$

we have $V(\mathbf{x}, \mathbf{y}) \geq 0$, with equality exactly at NE pairs of the game. One can show Ho95a, Har98 that $V(t):=V(\mathbf{x}(t), \mathbf{y}(t))$ satisfies $\dot{V}(t)=-V(t)$ along every solution of the differential inclusion (55). Hence the global attractor coincides with the set of equilibrium pairs.

For the special case of the matching pennies game with $n=2, a_{11}=a_{22}=1$ and $a_{12}=a_{21}=-1$, the above Lyapunov function reads $V(x, y)=|1-2 x|+|1-2 y|$, which is essentially the $\ell^{1}$ distance from the equilibrium $\left(\frac{1}{2}, \frac{1}{2}\right)$.

This result has recently been generalized to non-linear concave-convex zero-sum games HoSo02. There seems little hope to extend it beyond zero-sum games:

Conjecture $([$ Ho95a $])$. Let $(\mathbf{p}, \mathbf{q})>\mathbf{0}$ be an isolated interior equilibrium of a bimatrix game $(A, B)$, which is stable under the BR dynamics. Then $(A, B)$ is a c-zerosum game.

[Se00] and [Ber03] prove convergence to NE in $2 \times 3$, resp. $2 \times m$-games. For $n, m \geq 3$ limit cycles (Shapley polygons) were found in Sh64 and studied further in [Ro71], GaH95], KrS98, [FoY98]. A chaotic attractor for the BR dynamics has been constructed by Cowan Co92].

The best response dynamics for the symmetrized game has been studied in Ber01, Ber02], Cr03. The Wright manifold is no longer invariant. But in the cyclic $2 \times 2$-game (see (29)), all trajectories outside the line of NE converge to the unique Nash equilibrium on the Wright manifold (see Ber01]), which is a much stabler behaviour than the replicator dynamics (3) exhibits. A similar result holds if $(A, B)$ is a $c$-zerosum game; see Ber02.

3.4. Smoothed best replies. The BR dynamics can be approximated by smooth dynamics such as the logit dynamics

$$
\dot{x}_{i}=\frac{e^{a_{i}(\mathbf{x}) / \varepsilon}}{\sum_{j} e^{a_{j}(\mathbf{x}) / \varepsilon}}-x_{i}
$$

with $\varepsilon>0$. As $\varepsilon \rightarrow 0$, this converges to the best response dynamics, and every family of rest points $\hat{\mathbf{x}}(\varepsilon)$ accumulates in the set of Nash equilibria. There are (at least) two ways to motivate and generalize this 'smoothing'.

While $B R(\mathbf{x})$ is the set of maximizers of the linear function $\mathbf{z} \mapsto \sum_{i} z_{i} a_{i}(\mathbf{x})$ on $S_{n}$, consider $b_{\varepsilon v}(\mathbf{x})$, the unique maximizer of the function $\mathbf{z} \mapsto \sum_{i} z_{i} a_{i}(\mathbf{x})+\varepsilon v(\mathbf{z})$ on 
$\operatorname{int} S_{n}$, where $v$ is a strictly concave function int $S_{n} \rightarrow \mathbf{R}$ such that $\left|v^{\prime}(\mathbf{z})\right| \rightarrow \infty$ as $\mathbf{z}$ approaches the boundary of $S_{n}$. If $v$ is the entropy $-\sum z_{i} \log z_{i}$, the corresponding smoothed best response dynamics

$$
\dot{\mathbf{x}}=b_{\varepsilon v}(\mathbf{x})-\mathbf{x}
$$

reduces to (57) above FL98.

Another way to perturb best replies are stochastic perturbations. Let $\varepsilon$ be a random vector in $\mathbf{R}^{n}$ distributed according to some positive density function. For $\mathbf{z} \in \mathbf{R}^{n}$, let

$$
C_{i}(\mathbf{z})=\operatorname{Prob}\left(z_{i}+\varepsilon_{i} \geq z_{j}+\varepsilon_{j} \quad \forall j\right),
$$

and $\mathbf{b}(\mathbf{x})=C(\mathbf{a}(\mathbf{x}))$. It can be shown HoSa02 that each such stochastic perturbation can be represented by a deterministic perturbation as described before. The main idea is that there is a potential function $W: \mathbf{R}^{n} \rightarrow \mathbf{R}$, with $\frac{\partial W}{\partial a_{i}}=C_{i}(\mathbf{a})$ which is convex and has $-v$ as its Legendre transform. If the $\left(\varepsilon_{i}\right)$ are i.i.d. with the extreme value distribution $F(x)=\exp (-\exp (-x))$, then $C_{i}(\mathbf{a})=\frac{e^{a_{i}}}{\sum_{j} e^{a_{j}}}$ is the logit choice function and we obtain (57).

For the logit dynamics (57) and more generally (58), Lyapunov functions have been found for partnership games, zero-sum games and games with an interior ESS (see [Ho00]). Analogous results for bimatrix games are given in [HoH02].

An interesting class of games are the supermodular games (also known as games with strict strategic complementarities) [FT91] which are defined, with $a_{i, j}=\frac{\partial a_{i}}{\partial x_{j}}$, by

$$
a_{i+1, j+1}-a_{i, j+1}-a_{i+1, j}+a_{i, j}>0 \quad \forall i, j
$$

at every $\mathrm{x} \in S_{n}$. Stochastic dominance defines a partial order on the simplex $S_{n}$ :

$$
\mathbf{p} \succeq \mathbf{p}^{\prime} \quad \Leftrightarrow \quad \sum_{k=1}^{m} p_{k} \leq \sum_{k=1}^{m} p_{k}^{\prime} \quad \forall m=1, \ldots, n-1 .
$$

If all inequalities in (61) are strict, we write $\mathbf{p} \succ \mathbf{p}^{\prime}$. The pure strategies are totally ordered: $\mathbf{e}_{1} \prec \mathbf{e}_{2} \cdots \prec \mathbf{e}_{n}$.

The crucial property of supermodular games is the monotonicity of the best reply correspondence: If $\mathbf{x} \preceq \mathbf{y}, \mathbf{x} \neq \mathbf{y}$, then $\max B R(\mathbf{x}) \leq \min B R(\mathbf{y})$. This property was used in [Kr92] to prove convergence of fictitious play, and in HoSa02 the result was extended to perturbed best response maps.

Theorem 13 ([HoSa02]). For every supermodular game

$$
\mathbf{x} \preceq \mathbf{y}, \mathbf{x} \neq \mathbf{y} \quad \Rightarrow \quad C(\mathbf{a}(\mathbf{x})) \prec C(\mathbf{a}(\mathbf{y}))
$$

holds if the choice function $C: \mathbf{R}^{n} \rightarrow S_{n}$ is $C^{1}$ and the partial derivatives $C_{i, j}=$ $\frac{\partial C_{i}}{\partial a_{j}}$ satisfy for all $1 \leq k, l<n$

$$
\sum_{i=1}^{k} \sum_{j=1}^{l} C_{i, j}>0,
$$

and for all $1 \leq i \leq n$,

$$
\sum_{j=1}^{n} C_{i, j}=0
$$


The conditions (62), (63) on $C$ hold for every stochastic choice model (59), since there $C_{i, j}<0$ for $i \neq j$. As a consequence, the perturbed best response dynamics

$$
\dot{\mathbf{x}}=C(\mathbf{a}(\mathbf{x}))-\mathbf{x}
$$

generates a strongly monotone flow: If $\mathbf{x}(0) \preceq \mathbf{y}(0), \mathbf{x}(0) \neq \mathbf{y}(0)$, then $\mathbf{x}(t) \prec \mathbf{y}(t)$ for all $t>0$. The theory of monotone flows developed by Hirsch Hi88] and others (see [Sm95]) implies that almost all solutions of (64) converge to a rest point of (64).

3.5 The Brown-von Neumann-Nash dynamics. The Brown-von NeumannNash dynamics (BNN) is defined as

$$
\dot{x}_{i}=k_{i}(\mathbf{x})-x_{i} \sum_{j=1}^{n} k_{j}(\mathbf{x}),
$$

where

$$
k_{i}(\mathbf{x})=\max \left(0, a_{i}(\mathbf{x})-\mathbf{x}^{T} \mathbf{a}(\mathbf{x})\right)
$$

denotes the positive part of the excess payoff for strategy $i$. This dynamics is closely related to the continuous map f : $S_{n} \rightarrow S_{n}$ defined by

$$
f_{i}(\mathbf{x})=\frac{x_{i}+k_{i}(\mathbf{x})}{1+\sum_{j=1}^{n} k_{j}(\mathbf{x})},
$$

which Nash used (see Na51]) to prove the existence of equilibria by applying Brouwer's fixed point theorem: It is easy to see that $\hat{\mathbf{x}}$ is a fixed point of $\mathbf{f}$ iff it is a rest point of (65) iff $k_{i}(\hat{\mathbf{x}})=0$ for all $i$, i.e. iff $\hat{\mathbf{x}}$ is a Nash equilibrium of the game.

The differential equation (65) had been considered earlier by Brown and von Neumann [Br49], BrN50] in the special case of (linear) zero-sum games, for which they proved global convergence to the set of equilibria. This result was extended in Ni59, where generalizations of (65) were interpreted as price adjustment processes. While Nash's map (67) played a crucial role in economic equilibrium theory, 65) was revived as an evolutionary game dynamics by Skyrms Sk90 and studied further in [Sw93], Ber98], Ho00], Sa01]. Sandholm [Sa02 found a fascinating connection of generalizations of (65) to the regret-based learning models for repeated games of [HaM01].

Equation (65) defines an 'innovative better reply' dynamics. Indeed, strategies with payoff below average decrease in frequency, while strategies with payoff above average increase, as long as they are rare enough (and even if their frequency is 0 ). In contrast to the best response dynamics, (65) is Lipschitz (if payoffs are Lipschitz) and hence has unique solutions.

For linear games, a regular ESS is asymptotically stable, and an interior ESS is a global attractor; see [Ho00]. In partnership games, average payoff increases monotonically, and every orbit converges to the set of equilibria. We refer to [Ber98] for further results (especially on rock-scissors-paper games) and to [Sa02] for extensions to non-linear games. Interestingly, strictly dominated strategies can survive under this dynamics, as shown in BerH02.

We conclude with a result that summarizes the similar stability properties for all the game dynamics presented so far, in terms of (negative or positive) definiteness of the payoff matrix $A$. Recall $\mathbf{R}_{0}^{n}=\left\{\xi \in \mathbf{R}^{n}: \sum_{i} \xi_{i}=0\right\}$. 
Theorem 14. (i) If $\xi^{T} A \xi<0$ for all non-zero $\xi \in \mathbf{R}_{0}^{n}$ (i.e., the mean payoff function $\mathbf{x}^{T} A \mathbf{x}$ is strictly concave on $S_{n}$ ), then the game has a unique (symmetric) $N E \hat{\mathbf{x}}$. This $N E \hat{\mathbf{x}}$ is an ESS. $\hat{\mathbf{x}}$ is globally stable for the replicator dynamics (3), the best response dynamics (51), and the BNN dynamics (65).

(ii) If $\xi^{T} A \xi \leq 0$ for all $\xi \in \mathbf{R}_{0}^{n}$, then the set $E$ of $N E$ is convex. $E$ is stable for (3), and globally stable for (51) and (65). The perturbed dynamics (58) has a unique rest point $\hat{\mathbf{x}}(\varepsilon)$ for each $\varepsilon>0$. $\hat{\mathbf{x}}(\varepsilon)$ is globally stable for (58).

(iii) If $\xi^{T} A \xi>0$ for all non-zero $\xi \in \mathbf{R}_{0}^{n}$ (i.e., the mean payoff function $\mathbf{x}^{T} A \mathbf{x}$ is strictly convex on $S_{n}$ ) and there exists an interior $N E \hat{\mathbf{x}}$, then $\hat{\mathbf{x}}$ is globally repelling for (3) and locally repelling for (51) and (65). For small $\varepsilon>0$, the rest point $\hat{\mathbf{x}}(\varepsilon)$ near $\hat{\mathbf{x}}$ is locally repelling for (58).

This result follows for the replicator equation from [HoSi98], and for the other dynamics from [Ho00, Hop99b and BerH02]. It can be extended to non-linear payoff functions (for BNN see [Sa02]). Zero-sum games which satisfy (ii) lie on the border between stability and instability. An instructive special case is the rockscissors-paper game (91) with cyclic symmetry $\left(a_{i}=a, b_{i}=b\right)$. For $a<b, a=b$, and $a>b$ this game belongs to the cases (i), (ii), and (iii), respectively. The intuitive reason why for $a>b$ the unique equilibrium $\hat{\mathbf{x}}$ is unstable for every reasonable evolutionary dynamics is that at $\hat{\mathbf{x}}$ the population earns in this case less than along the best reply cycle $\mathbf{e}_{1} \rightarrow \mathbf{e}_{2} \rightarrow \mathbf{e}_{3} \rightarrow \mathbf{e}_{1}$.

3.6 The adjustment property. The adjustment property, introduced in Sw93, is defined by

$$
\dot{\mathbf{x}}^{T} \mathbf{a}(\mathbf{x}) \geq 0
$$

in $S_{n}$, with strict inequality whenever $\mathbf{x}$ is not a Nash equilibrium (or a rest point of the replicator dynamics). Any vector field on $S_{n}$ satisfying this property is said to be a myopic adjustment dynamics (MAD). This means that the population always moves towards a better reply to the present state - arguably, a minimal requirement for any adaptation worth its name. All dynamics considered so far, except those in section 3.4 of course, satisfy the adjustment property (for monotone selection dynamics, see [Ho95b], FL98]).

For myopic adjustment dynamics, a strict NE is always asymptotically stable. Interestingly, if $\hat{\mathbf{x}}$ is an interior equilibrium, then the dynamics heading straight towards it, i.e. $\dot{\mathbf{x}}=\hat{\mathbf{x}}-\mathbf{x}$, belongs to the MAD class iff $\hat{\mathbf{x}}$ is an ESS. For a partnership game, the mean payoff $\mathbf{x}^{T} A \mathbf{x}$ increases along orbits [Ho95b], Sa01].

However, as simple counterexamples show, the definite stability and instability results in the previous theorem do not apply to myopic adjustment dynamics in general. Indeed, for every linear game with a unique NE $\mathbf{p}$ one can construct myopic adjustment dynamics having $\mathbf{p}$ as the global attractor.

Hence the question arises whether there is an adjustment dynamics that always (i.e., for every game, from every initial condition) converges to equilibrium. The answer is no: there exist one-parameter families of games, each having a unique, interior Nash equilibrium $\mathbf{p}$, such that for every MAD depending continuously on the game, there is an open set of parameters for which an open set of orbits does not approach p ([HoSw96]; see also [HoSi98]).

This shows that the non-convergence of orbits to NE is not a weakness of this or that particular dynamics, but that there are situations where no evolutionary 
approach can be reduced to an equilibrium analysis. Every 'reasonable' dynamic approach leads to regular or irregular cycling behaviour for certain games.

\section{Extensions}

4.1. Population genetics. The first applications of evolutionary games concerned animals without the cognitive capacities to imitate and to learn (see [MS74, [MS82]). Their behavioural programs were inherited. The original motivation of the replicator dynamics was 'like begets like': the different types were supposed to breed true. This, of course, assumes clonal replication, which holds only for very simple organisms.

In sexually reproducing populations, individuals inherit their genes from both parents. Let us consider one gene locus where the alleles (that is, the different types of genes) $A_{1}, \ldots, A_{N}$ can occur. An individual's genotype is then described by the pair $\left(A_{i}, A_{j}\right)$, where the first element denotes the allele inherited from the father and the second the allele inherited from the mother. We denote the frequencies of the alleles in the population by $x_{1}, \ldots x_{N}$, so that the state of the 'gene pool' is described by $\mathbf{x} \in S_{N}$. Usually, one assumes that as a consequence of random mating, the population is in Hardy-Weinberg equilibrium; i.e. the frequency of the gene pair $\left(A_{i}, A_{j}\right)$ is given by $x_{i} x_{j}$. One also assumes that the genotypes $\left(A_{i}, A_{j}\right)$ and $\left(A_{j}, A_{i}\right)$ lead to the same phenotype, i.e. that genes act independently of whether they were transmitted maternally or paternally. If the 'fitness' of genotype $\left(A_{i}, A_{j}\right)$ (for instance, the survival rate of this genotype) is given by a constant $a_{i j}$ which is independent of the allelic frequencies $x_{i}$, one obtains the replicator equation (3) with a symmetric matrix $A$. This can be viewed as a partnership game played by the alleles in the gene pool.

The case of frequency dependent selection leads to more complex game dynamics (see, e.g., [MS81, Es82, Cr92, Hi94, HoSi98]). Let us assume that an individual with genotype $\left(A_{i}, A_{j}\right)$ uses a strategy $\mathbf{p}(i j) \in S_{n}$ for a population game described by the $n \times n$-matrix $A$ (with $\mathbf{p}(i j)=\mathbf{p}(j i)$ ). The mean strategy $\mathbf{p} \in S_{n}$ in the population is given by

$$
\mathbf{p}=\mathbf{p}(\mathbf{x})=\sum_{i j} x_{i} x_{j} \mathbf{p}(i j) .
$$

Since a given allele $A_{i}$ belongs with probability $x_{j}$ to an $\left(A_{i}, A_{j}\right)$ - or an $\left(A_{j}, A_{i}\right)$ individual, the expression

$$
\mathbf{p}^{i}=\sum x_{j} \mathbf{p}(i j)=\frac{1}{2} \frac{\partial \mathbf{p}}{\partial x_{i}}
$$

yields the frequencies of the strategies used by allele $A_{i}$. The usual ansatz then leads to

$$
\dot{x}_{i}=x_{i}\left[\left(\mathbf{p}^{i}-\mathbf{p}\right)^{T} A \mathbf{p}\right]
$$

on $S_{N}$, which describes a frequency-dependent selection dynamics in the gene pool.

If the replicator equation (3) is a Shahshahani gradient, then so is (71) [Si87. In particular, if $n=2$, i.e. if there are only two strategies, then the potential of (71) is given by

$$
V(\mathbf{x})=\frac{\alpha}{2}\left[\sum x_{i} x_{j} p_{1}(i j)-\frac{a_{22}-a_{12}}{\alpha}\right]^{2}
$$


provided $\alpha:=a_{11}-a_{21}+a_{22}-a_{12} \neq 0$. If the $2 \times 2$-game admits a mixed ESS $\hat{\mathbf{p}}$, i.e. if $a_{11}-a_{21}$ and $a_{22}-a_{12}$ are both negative, then the strategy mix $\mathbf{p}$ converges to the ESS, provided

$$
S(\hat{\mathbf{p}}):=\left\{\mathbf{x} \in S_{N}: \mathbf{p}(\mathbf{x})=\hat{\mathbf{p}}\right\}
$$

is non-empty.

Let us define the $n \times n$ matrix $C(\mathbf{x})$ as the covariance matrix of the allelic strategies $\mathbf{p}^{i}$, i.e.

$$
c_{k l}(\mathbf{x})=\sum x_{i}\left(p_{k}^{i}-\mathbf{p}_{k}\right)\left(p_{l}^{i}-\mathbf{p}_{l}\right) .
$$

Then the frequencies $\mathbf{p}$ of the strategies in the population satisfy

$$
\dot{\mathbf{p}}=2 \sum \mathbf{p}^{i} \dot{x}_{i}=2 C(\mathbf{x}) A \mathbf{p} .
$$

If at some $\mathbf{x} \in \operatorname{int} S_{N}$ one has $\dot{\mathbf{p}}=\mathbf{0}$, then $\dot{\mathbf{x}}=\mathbf{0}$. Thus if the strategy in the population does not change, then the composition in the gene pool does not change.

The state $\hat{\mathbf{p}} \in S_{n}$ is said to be strategically stable if (a) $S(\hat{\mathbf{p}})$ consists of rest points of (71) and (b) for each neighborhood $U$ of such an $\hat{\mathbf{x}} \in S(\hat{\mathbf{p}})$ there is a neighborhood $V$ of $\hat{\mathbf{x}}$ such that for all $\mathbf{x} \in V$, one has $\mathbf{x}(t) \in U$ for all $t>0$ and $\mathbf{p}(\mathbf{x}(t)) \rightarrow \hat{\mathbf{p}}$ for $t \rightarrow+\infty$. (Property (b) is weaker than asymptotic stability but stronger than stability.)

Theorem 15 (CrHH96). For $n=2$ and $n=3$, if $\hat{\mathbf{p}}$ is an ESS and $S(\hat{\mathbf{p}})$ is non-empty, then $\hat{\mathbf{p}}$ is strategically stable.

The proof makes heavy use of center manifold calculations. For $n \geq 4$ the problem is still open. However, one has the generic result

Theorem 16 ([Cr92, HoS88, HoSi98]). If $\hat{\mathbf{p}} \in \operatorname{int} S_{n}$ is an ESS, then it is strategically stable, provided that for all $\hat{\mathbf{x}} \in S(\hat{\mathbf{p}})$ the covariance matrix $C(\hat{\mathbf{x}})$ has full rank (the minimum of $N-1$ and $n-1$ ).

Several authors have studied the effects of mutation and recombination on the long-term evolution of strategies; see e.g. [Es96, [Ha96], Wei96]. For other, fertility-based, models at the interface of genetics and game theory, we refer to HoSS82.

4.2 Continua of strategies and adaptive dynamics. So far we have considered games with a finite number of pure strategies. In many cases, one encounters games where the pure strategies belong to a continuum. This could be, for instance, the size of an investment or the aspiration level of a player. In biology, such strategies correspond to some continuously varying trait - the size of antlers, for instance, the sex ratio in a litter or the virulence of an infection. A particularly interesting application concerns the 'war of attrition game'; see [MS82].

There exist several approaches to modelling the corresponding population dynamics. We start by describing that of Bomze (see [Bo90], [Bo91]). The set of strategies is described, not by $\{1, \ldots, n\}$, but by a possibly infinite set $S \subset \mathbf{R}^{n}$; a population corresponds to a probability measure on the Borel subsets of $S$; the metric is given by the variational norm on the Banach space of signed measures on $S$ and the time-evolution $t \rightarrow Q(t)$ of the population obeys an ordinary differential equation in this Banach space which corresponds to the replicator equation, namely

$$
\dot{Q}(A)=\int_{A} \sigma(\mathbf{x}, Q) d Q
$$


for all Borel subsets $A$ of $S$, where $\sigma(\mathbf{x}, Q)$ is the difference between the payoff for using strategy $\mathrm{x} \in S$ in a population $Q$ and the average payoff in population $Q$. Under mild conditions on the payoff function, this dynamics is well defined. Interestingly, strict NE need not be stable; see OR01]. But uninvadable populations are stable and weakly attracting in the sense that those states which are close-by (in the variational norm) converge weakly (i.e., in distribution). ( $P$ is uninvadable if there is an $\epsilon>0$ such that for all $Q$, the state $P$ does better than $Q$ against all mixtures of $(1-\eta) P+\eta Q$ with $\eta<\epsilon$. This $\epsilon$ plays the role of a uniform invasion barrier; for games with infinitely many strategies, it need not exist for every ESS.) For interesting applications and extensions of this approach see [BoB95, OR01. Stability results with respect to weak convergence are related to the CSS concept discussed below; see [EsMS97] and OR02]. Similar results are found by HoOR03. for the analog of the BNN dynamics.

Faced with a continuum of pure strategies, modellers frequently make up for the increased size of the strategy space by simplifying the population structure. An example is the adaptive dynamics approach, where it is assumed that the population is essentially homogenous, all individuals sharing the same strategy or trait-value, with the exception of an occasional, tiny minority adopting a strategy which is close to the prevailing strategy. The basic question then is whether such dissidents can invade the resident population. Recall that according to the intuition behind the concept of an ESS, an evolutionarily stable strategy cannot be invaded.

Let us first consider the case that the resident strategy is given by some real number $s$ and the invading strategy by $s+h$ (with $h$ small). The payoff for an individual using strategy $s+h$ in a population where almost all individuals use $s$ is given by $A(s+h, s)$. The invader succeeds if $W(h, s):=A(s+h, s)-A(s, s)$ is positive. The adaptive dynamics [HoS90] is given by the differential equation

$$
\dot{s}=\frac{\partial W}{\partial h}(0, s) \text {. }
$$

Depending on whether $\dot{s}$ is positive or negative, this means that dissidents with a larger (resp. smaller) $s$-value can invade. A strategy $\hat{s}$ is a local strict Nash equilibrium if $W(h, \hat{s})<0$ for all small $h \neq 0$. It is said to be convergence-stable if for all $s \neq \hat{s}$ in a neighborhood of $\hat{s}$, the difference $W(h, s)$ has the sign of $h(\hat{s}-s)$ for small $h$ [Tay89], [Es96], [Le90]. The point $\hat{s}$ is a rest point of (77) if it is a local strict NE or convergence-stable. Generically, a rest point $\hat{s}$ is convergence-stable iff $\frac{\partial^{2} W}{\partial s \partial h}(\hat{s})<0$, and a local strict Nash equilibrium iff $\frac{\partial^{2} W}{\partial^{2} h}(\hat{s})<0$. Obviously, if the (homogenous) population is in a state $s$ close to a convergence-stable $\hat{s}$, then small deviations in the direction towards $\hat{s}$ will succeed. Interestingly, the same need not hold if $\hat{s}$ is a local strict Nash equilibrium, although this is a local ESS. In this sense (as pointed out by Es83, No90, TaKi91]), an ESS can be unattainable (and 'evolutionary stability' therefore is a misnomer): there exist strategies $\hat{s}$ such that (a) if all members of the population adopt $\hat{s}$, dissidents cannot invade, but (b) populations which are close-by will evolve away from $\hat{s}$. The corresponding states of the population have been termed 'Garden of Eden'configurations. Conversely, there exist states attracting near-by populations, but invasible by dissidents. States which are both local strict NE and convergence-stable have been termed continuously stable strategies (or CSS) by Eshel [Es83].

In the $n$-dimensional case, let us consider a population where all individuals share the same strategy, given by an s in some open $U \in R^{n}$. A 'mutant' individual with 
trait $\mathbf{s}+\mathbf{h}$ would obtain an expected payoff $A(\mathbf{s}+\mathbf{h}, \mathbf{s})$, and its relative advantage (compared with the resident population) is $W(\mathbf{h}, \mathbf{s}):=A(\mathbf{s}+\mathbf{h}, \mathbf{s})-A(\mathbf{s}, \mathbf{s})$. In this case the vector field $D_{\mathbf{h}} W(\mathbf{h}, \mathbf{s})$, evaluated for $\mathbf{h}=\mathbf{0}$, points in the direction of the maximal increase of the advantage experienced by a potential mutant. If the vector does not vanish at the point $\mathbf{s}$, it defines a half-space with the property that a minority with strategy $\mathbf{s}+\mathbf{h}$ close to $\mathbf{s}$ will invade if and only if the strategy lies in that half-space. The corresponding orbits of this vector field offer a rough approximation of the evolution of a homogenous population by mutation and selection, where mutations are small and occur so rarely that their fate (elimination, or else fixation in the population) is settled before the next mutation occurs [DiLa96, [Me96], Ge02. This adaptive dynamics has been intensively used in mathematical models of the theory of evolution, including speciation, co-evolution, etc.

In a more general setup, one can assume that genetic, developmental or other constraints render variation in some directions more likely than in others. This can be described by a Riemannian metric on a submanifold $S \subseteq \mathbf{R}^{n}$ associating to each $\mathbf{s} \in S$ a symmetric positive definite matrix $G(\mathbf{s})$ such that the inner product in $T_{\mathbf{s}} S$, the tangent space at $\mathbf{s}$, is given by

$$
[\boldsymbol{\eta}, \boldsymbol{\xi}]_{\mathbf{s}}=\boldsymbol{\eta}^{T} G(\mathbf{s}) \boldsymbol{\xi} .
$$

The adaptive dynamics is then described by the vector field $\dot{\mathbf{s}}$ satisfying

$$
[\boldsymbol{\eta}, \dot{\mathbf{s}}]_{\mathbf{s}}=D_{\mathbf{y}} A(\mathbf{y}, \mathbf{s})(\boldsymbol{\eta})
$$

for all $\boldsymbol{\eta}$ in the tangent space at $\mathbf{s}$, where the derivative is evaluated at $\mathbf{y}=\mathbf{s}$.

In particular, if the state space is int $S_{n}$ and $A(\mathbf{y}, \mathbf{x})$ is linear in $\mathbf{y}$, i.e. of the form $\mathbf{y}^{T} \mathbf{a}(\mathbf{x})$, one obtains

$$
\dot{x}_{i}=\sum c_{i j}(\mathbf{x}) a_{j}(\mathbf{x})
$$

where

$$
C=G^{-1}-\left(\mathbf{g}^{T} \mathbf{1}\right)^{-1} \mathbf{g g}^{T}
$$

and $\mathbf{g}=G^{-1} \mathbf{1}$ (see [HoSi98]; the same equation has also been derived from a learning model in Hop99a). In the special case of the Shahshahani metric, i.e. with $g_{i j}(\mathbf{x})=\delta_{i j} \frac{1}{x_{i}}$, where $\delta$ is the Kronecker delta, (80) yields the replicator equation (37).

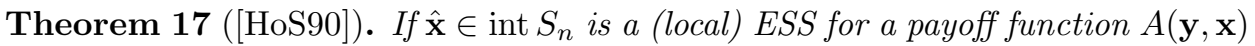
which is linear in $\mathbf{y}$, then $\hat{\mathbf{x}}$ is asymptotically stable for each adaptive dynamics (80).

Even in the one-dimensional case, this need not hold in the absence of the linearity assumption; see [Me01].

4.3 Discrete time dynamics and fictitious play. The equivalent of the replicator equation (3) in discrete time $t=0,1,2, \ldots$ is

$$
x_{i}(t+1)=x_{i}(t) \frac{(A \mathbf{x}(t))_{i}+c}{\mathbf{x}(t)^{T} A \mathbf{x}(t)+c}
$$

with some constant $c$ whose task is to ensure that all numerators (and hence also the denominator) are positive. This constant can be viewed as a 'background payoff' and accordingly the values $a_{i j}$ as (positive or negative) increments in payoff. The resulting discrete dynamical system on $S_{n}$ still satisfies the folk theorem (Nash equilibria are rest points, etc.), but for $n>2$ it can offer more complex behaviour 
than the continuous time dynamics. (Such deviations can be tuned down by increasing c). At least, (82) is a diffeomorphism on $S_{n}$; see [LoA83]. For zero-sum games, interior equilibria are globally repelling [AL84, and ESS need no longer be asymptotically stable. The discrete dynamics of the rock-scissors-paper game is still simple in the case of cyclic symmetry Ho84, but in general it can exhibit attracting closed invariant curves in int $S_{3}$, as shown in Figure 4 (see Wei91]). Dominated strategies can survive under (82) (see [DeS92]) but are still eliminated

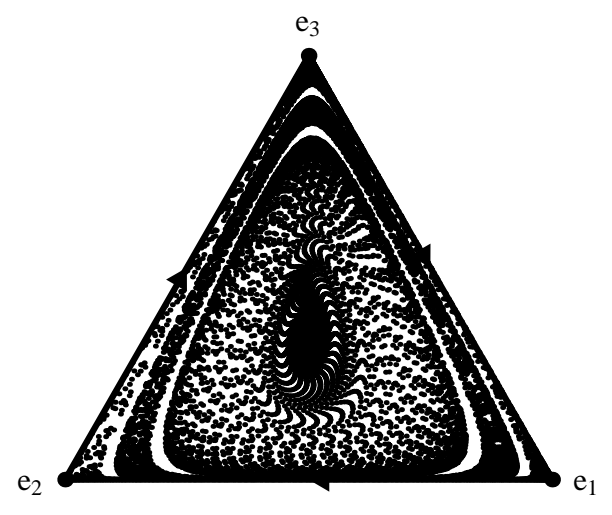

Figure 4. The discrete time replicator dynamics (82) for the rockscissors-paper game with payoff matrix $A=\left(\begin{array}{ccc}0 & 4 & -2 \\ -2 & 0 & 4 \\ 13.75 & -11.75 & 0\end{array}\right)$ and $c=13$.

under the alternative discrete dynamics CaS92

$$
x_{i}(t+1)=x_{i}(t) \frac{e^{(A \mathbf{x})_{i}}}{\sum_{k} x_{k} e^{(A \mathbf{x})_{k}}}
$$

which is occasionally better behaved than (82). For instance, if $\mathbf{p}$ is a unique NE in int $S_{n}$, and the $\omega$-limit of $\mathbf{x}(t)$ is disjoint from $\operatorname{bd} S_{n}$, then the time average $(\mathbf{x}(1)+\cdots+\mathbf{x}(N)) / N$ converges to $\mathbf{p}$, just as in (11). The permanence condition (13) still implies permanence of (83), but for (82) only for large $c$; see GH03.

Certain models of reinforcement learning lead to stochastic processes in discrete time whose expected motion is closely related to the replicator equation; see [BöS97, [Po97], [ErR98, [Ru99], Bö00, [LaTW01], Hop02, Beg02 and HopP02.

A discrete-time equivalent of the best response dynamics is given by the fictitious play process of Brown and Robinson ( $\mathrm{Br} 49, \mathrm{Br} 51, \mathrm{Ro51})$. In a population setting appropriate for evolutionary game dynamics, this could be viewed as a process where, at every time step, a new player enters the population and adopts (once and for all) a strategy which is a best reply to the current state $\mathbf{x}(t)$; see [Ho95a]. This yields

$$
\mathbf{x}(t+1)=\frac{1}{t+1} B R(\mathbf{x}(t))+\frac{t}{t+1} \mathbf{x}(t)
$$

The original setting of the fictitious play (FP) procedure of Brown Br51 is that of an asymmetric game played repeatedly by the two players, who both choose in 
each round a best reply to the average of the strategies used by the co-player in the previous rounds. Thus

$$
\mathbf{p}_{t+1} \in B R\left(\mathbf{Q}_{t}\right), \quad \mathbf{q}_{t+1} \in B R\left(\mathbf{P}_{t}\right)
$$

with initial values $\mathbf{p}_{1}=\mathbf{P}_{1} \in S_{n}, \mathbf{q}_{1}=\mathbf{Q}_{1} \in S_{m}$ and

$$
\mathbf{P}_{t}=\frac{1}{t} \sum_{k=1}^{t} \mathbf{p}_{k}, \quad \mathbf{Q}_{t}=\frac{1}{t} \sum_{k=1}^{t} \mathbf{q}_{k},
$$

or equivalently

$$
\mathbf{P}_{t+1}-\mathbf{P}_{t} \in \frac{1}{t+1}\left[B R\left(\mathbf{Q}_{t}\right)-\mathbf{P}_{t}\right], \quad \mathbf{Q}_{t+1}-\mathbf{Q}_{t} \in \frac{1}{t+1}\left[B R\left(\mathbf{P}_{t}\right)-\mathbf{Q}_{t}\right] .
$$

The following result was conjectured in [Br51] and proved in [Ro51].

Theorem 18. For zero-sum games, any such sequence of mixed strategies $\left(\mathbf{P}_{t}, \mathbf{Q}_{t}\right)$ converges to the set of equilibrium pairs (i.e. maximin solutions).

A modern proof runs as follows [Ho95a], Har98, [HoSo02]: A fictitious play path (86) is an Euler discretization sequence with diminishing step sizes. The set of its limit points is a (weakly) invariant set of the best response dynamics; i.e., it consists of complete orbits of (55). Hence the global attractor of (55) contains all limit points of FP paths. For zero-sum games, the global attractor is the set of NE; see section 3.3 .

Fictitious play converges also for partnership games MoSh96b], and for certain supermodular games [Kr92].

The results on the smoothed best response dynamics can be applied to stochastic fictitious play [FL98] where $\left(\mathbf{P}_{t}, \mathbf{Q}_{t}\right)$ from (85)), (86) describes a stochastic process, with (perturbed) best replies chosen according to (59),

$$
\operatorname{Prob}\left(\mathbf{p}_{t+1}=\mathbf{e}_{i} \mid \mathbf{Q}_{t}=\mathbf{y}\right)=C_{i}(A \mathbf{y}) .
$$

The expected increments are given by

$$
E\left(\mathbf{P}_{t+1}-\mathbf{P}_{t} \mid \mathbf{Q}_{t}=\mathbf{y}\right)=\frac{1}{t+1}[C(A \mathbf{y})-\mathbf{y}],
$$

with a similar expression for the second player. Stochastic approximation theory Ben99 then allows us to derive convergence results for this stochastic process (see HoSa02]), which are analogous to results for the smoothed best response dynamics and extend previous results of [BenH99], [FL98 and others.

Of course one can also study discrete time dynamics in the context of population genetics - this is particularly appropriate for populations with disjoint generations. Equation (82) with symmetric $A$ (corresponding to a partnership game) is nothing but the classical one-locus selection equation of population genetics. The average payoff $\mathbf{x}^{T} A \mathbf{x}$ increases along all orbits, and each orbit converges to a rest point. This equation describes the scenario where each genotype has a 'fitness', or survival probability, which is independent of the composition of the population. If the genotype specifies a strategy whose success depends on the co-players' behaviour, the resulting frequency-dependent selection is much more complex. If there are only two strategies, however, with payoffs $s_{1}$ and $s_{2}$ depending on the frequencies of the $N$ alleles in the gene pool, then $\left(s_{1}-s_{2}\right)^{2}$ is a strict Lyapunov function and the difference $s_{1}-s_{2}$ converges to 0 without changing sign ([Le84] and [HoSi98]). 
Similar discrete time dynamics can be studied for bimatrix games. For the cyclic $2 \times 2$-game, for instance, the interior equilibrium is globally repelling for the analog of (82), but for imitation dynamics where more than one individual is observed, attracting invariant curves may surround the unstable equilibrium; see HoSc00. The following discrete time version suggested in [Ho96] looks particularly interesting:

$$
\begin{gathered}
x_{i}(t+1)=x_{i}(t) \frac{(A \mathbf{y}(t))_{i}+c}{\mathbf{x}(t)^{T} A \mathbf{y}(t)+c} \\
y_{j}(t+1)=y_{j}(t) \frac{\left(B^{T} \mathbf{x}(t+1)\right)_{j}+d}{\mathbf{y}(t)^{T} B^{T} \mathbf{x}(t+1)+d} .
\end{gathered}
$$

(Note the time $t+1$ for the $\mathbf{x}$ in the second equation: the two players here alternate their moves.) Such a map is still volume-preserving, and its eigenvalues are those of a symplectic map; hence it shares the conservative properties of (21)-(22).

4.4 Diffusion models. Let us consider now populations of players distributed in a continuous space and described by the spatial density $\mathbf{p}(x, t) \in S_{n}$, where $x$ denotes the space variable and the $p_{i}$ are the frequencies of the strategies. If the local interaction among the players is described by some dynamics $\dot{\mathbf{p}}=\mathbf{f}(\mathbf{p})$, and the random motion of the players can be modelled by diffusion, this yields a reaction-diffusion equation

$$
\frac{\partial \mathbf{p}}{\partial t}=\mathbf{f}(\mathbf{p})+d \frac{\partial^{2} \mathbf{p}}{\partial x^{2}} .
$$

Here $d>0$ is the diffusion constant, which for simplicity we assume to be independent of the strategy $i$. (If this diffusion constant depends on the strategy, then one has to interpret $\mathbf{p}(x, t)$ as the spatial density of the numbers - instead of the frequencies - of players at place $x$ at time $t$; see [Vi89, [HuVi92, CrVi97]. In this case Turing instabilities may occur: NE that are asymptotically stable under the reaction dynamics $\dot{\mathbf{p}}=\mathbf{f}(\mathbf{p})$ may become unstable in the reaction-diffusion dynamics. However, gratifyingly, an ESS cannot be destabilized under the replicator dynamics with diffusion; see [Vi89], CrVi97].)

Suppose now that space is the real line, and consider the bistable game $A=\left(\begin{array}{ll}a & 0 \\ 0 & b\end{array}\right)$ with $a, b>0$. Then (91) reduces to a well-studied equation (see [Fi79])

$$
\frac{\partial p}{\partial t}=p(1-p)((a+b)-b p)+d \frac{\partial^{2} p}{\partial x^{2}} .
$$

There exists a travelling wave $p(x, t)=U(x-c t)$, where $U: \mathbf{R} \rightarrow(0,1)$ is strictly increasing, with $U(-\infty)=0, U(+\infty)=1$, and the wave speed $c$ is $a-b$, up to a positive factor. Hence, for $a>b$ the strict equilibrium $\mathbf{e}_{1}$ with the higher payoff $a$ supersedes the other equilibrium. Furthermore, $\mathbf{e}_{1}$ is spatially dominant in the following sense (see [Fi79]): its basin of attraction contains an open set in the compact-open topology. If initially the population is close to $\mathbf{e}_{1}$ on a sufficiently large (but finite) interval, then it will converge to $\mathbf{e}_{1}$ for all $x$.

This result for $2 \times 2$ games is fairly independent of the specific reaction dynamics: it also holds for BR, BNN, and some other adjustment dynamics. For systems of reaction-diffusion equations, no general results on spatial dominance seem to be known. In particular, for (91) with $n \geq 3$ strategies there are only few results, 
apart from the two obvious facts that every game has at most one spatially dominant equilibrium and that many games have no spatially dominant equilibrium.

Consider now a $2 \times 2$ bimatrix game, with payoff matrices normalized to $A=$ $\left(\begin{array}{cc}a_{1} & 0 \\ 0 & b_{1}\end{array}\right)$, and $B=\left(\begin{array}{cc}a_{2} & 0 \\ 0 & b_{2}\end{array}\right)$, where $a_{i}, b_{i}>0$. It has two strict NE, namely $\mathbf{e}_{i}=\left(\mathbf{e}_{i}, \mathbf{f}_{i}\right)$ $(i=1,2)$. The following has been shown in [Ho97, Ho99]:

Theorem 19. The equilibrium $\mathbf{e}_{1}$ is spatially dominant for the bimatrix version of (91) with BR dynamics if

$$
a_{1} a_{2}>b_{1} b_{2} .
$$

Furthermore, there exists a unique monotone travelling wave that connects the two strict NE. Its wave speed $c$ is positive; i.e., $\mathbf{e}_{1}$ supersedes $\mathbf{e}_{2}$.

The condition (93) has been introduced by [HaSe88] as a criterion to select $\mathbf{e}_{1}$ over $\mathbf{e}_{2}$ and is known as risk-dominance. There is a simple axiomatic characterization for it, which is related to the fact that $\mathbf{e}_{1}$ has a larger basin of attraction than $\mathbf{e}_{2}$ under the best response dynamics.

With the replicator dynamics as reaction term, the spatially dominant equilibrium is - in contrast to (92) - not determined by a simple condition such as (93); see HoHV97.

Most other results about spatial dominance concern $N$ person games played between $N \geq 3$ distinct populations which are outside the scope of this survey. We mention only unanimity games, which are a direct generalization of the bimatrix game above to $N$ players: If all players unanimously use the first (second) strategy, player $i$ gets payoffs $a_{i}>0$ (resp. $b_{i}>0$ ). All other payoffs are 0 . In analogy with (93), HaSe88] postulated

$$
\prod_{i=1}^{N} a_{i}>\prod_{i=1}^{N} b_{i}
$$

as selection criterion for $\mathbf{e}_{1}$. Ho99] shows that (94) indeed implies the spatial dominance of $\mathbf{e}_{1}$. So far this seems to be, for $N>2$, the only known evolutionary justification of Nash products as a selection criterion.

For related models and equilibrium selection results for discrete space (grids or networks as described below), see e.g. [B193], [E193], Mor00], Kos02].

4.5 Lattice-based populations. Another much studied approach to spatial games is based on a more detailed modelling of the networks of interacting players. In the simplest case, players are assumed to be located at the vertices of a given lattice. At each of the (discrete) time steps $t$, each individual $k$ engages in pairwise interactions with each of the co-players $l$ from some neighborhood $N(k)$. Each game yields a payoff $P(k, l)$, and player $k$ 's total payoff is $P(k):=\sum_{l \neq k \in N(k)} P(k, l)$. Then, players update their strategies through some imitation rule. For instance, player $k$ could compare payoffs with all neighbors $l \in N(k)$ (who, in turn, have interacted within their neighborhoods $N(l)$ ) and adopt the strategy of that player who did best (using a tie-breaking rule if the outcome is not uniquely specified). Again, many variants are possible: in particular, the set of $k$ 's potential role models could be distinct from $N(k)$, the imitation rule could be stochastic rather than deterministic, the updating of the strategy could occur at different moments for different players, the neighborhood lattice could evolve in time, etc. In addition to the strategic interaction and the updating, which reflects some kind of selection 
of strategies, one could include migration, mutation, recombination and extinction phenomena. Depending on the details, the resulting process describing the evolution of the distribution of strategies over the lattice can be stochastic (see BH87, NoBoMay94, Du99, Kos02]) or result in a deterministic cellular automaton, as in NoMay92, [NoMay93]. Such spatial games have given rise to few theorems, except for one-dimensional lattices (see [B193, EsSS98, Kos02), but offer a promising field for experimental mathematics suggesting many conjectures.

It may happen that strategies which are strictly dominated can resist elimination and survive, for instance by freezing into clusters or by moving across the lattice in a kind of spatio-temporal chaos; see [NoMay92]. More generally, the neighborhood structure usually tends to favor the long-term co-existence of strategies which would not co-exist in well-mixed populations. Specific initial conditions can reproduce in neighboring locations and travel like 'gliders' across the lattice. Often, almost all initial conditions seem to lead to the same overall average frequency NoMay92. The relationship between the evolution of the strategies on the lattice and the corresponding mean field dynamics, e.g. the replicator equation applied to the spatial averages of the strategies' frequencies, offers a tantalising field of open problems Du99.

4.6 Finite populations. In finite populations of size $N$, some of the concepts of evolutionary game dynamics must be modified. In particular, the relevance of interior ESS has been debated (cf. [MS88], [S88] and the literature therein). Invasion barriers below $1 / N$ make little sense; and since the frequencies change in finite steps, it may happen that if a player switches towards a better strategy, this strategy ceases to be better. Schaffer's [S88] reformulation of the ESS concept for a 'playing the field' situation with $N$ players is a useful framework for economic applications; see S89, AF01 and AF02. The stability of mixed NE in a random matching-like setting is studied in Oe97 and AF00.

For finite populations, stochastic models often seem more realistic than deterministic ones. The paper by Foster and Young [FoYo90, who simply added white noise to the replicator equation, inspired a whole literature of stochastic models, all using and adapting large deviation techniques from [FrW84, each postulating different behavior at the individual level. Young Y93, YY98 considers a truncated stochastic variant of fictitious play. Another model [KaMR93] incorporates Darwinian ideas (i.e., successful strategies spread) via an imitation process where agents copy the most successful strategies, whereas [KaR95] considers myopic best reply. This makes a difference; see [Sa98]. Here the population is assumed to be finite, and in each period, one or several players can adjust by imitating the best or choosing a best reply to the current state. In addition, random perturbations occurring with a small probability $\varepsilon$ can alter the strategy of every player. This defines a Markov process describing the number of players using each strategy, $i=1, \ldots, n$. The random perturbations guarantee that the process is irreducible and hence admits a unique stationary distribution. The limit of this distribution, for $\varepsilon \rightarrow 0$, is a well defined distribution which approximates the time average spent in the different states. The support of this distribution is said to be the set of long run equilibria. This set can be a cycle, but for important classes of games, it consists of a single NE. 
For $2 \times 2$ coordination games most of these models select the risk dominant equilibrium, but see [Sa97] (a summary of contributions by Samuelson and coworkers on this topic) and [V96] for interesting exceptions. For larger games also, the differences between the 'long run equilibrium' of [KaR95] and the 'stochastically stable equilibrium' of YY93] become pronounced; see [JJS99.

This approach has led to many important economic applications, such as oligopolies [V97, RS01, [AFS00], signalling games [NS97], and insurance markets ATW02, to mention a few.

Finite population models in the form of Markov chains also provide microfoundations for many of the differential equations considered in sections 2 and 3 ; see BenW00, HoSa01, Sch01] for rigorous results connecting the large population limit with the asymptotic behavior of the differential equations. This branch of stochastic approximation theory relies heavily on the study of invariant measures.

4.7 Applications. In this expository paper, we aim at stressing the variety of plausible dynamics which describe adaptive mechanisms underlying game theory. The replicator equation describes just one of many dynamics. For some models of evolutionary game theory, it does not suffice to specify the strategies and the payoff values; one must also be explicit about the transmission mechanisms describing how strategies spread within a population. If strategies spread through inheritance, this is straightforward (although we are presently still far from a complete understanding of how a given behavioural trait is related to the genome). Most interesting examples of strategies spread through learning, but again, the details of such a process are likely to be considerably more complex than the rules imitate the better or proportional imitation. As for the best reply and related dynamics, they probably demand more cognitive capabilities than most individuals can muster. Fortunately, for many games, most dynamics lead to the same predictions.

We do not try to describe the wealth of possible applications of evolutionary game theory; all we attempt here is to give some signposts to the huge relevant literature.

The first applications of, and indeed the motivation for, evolutionary game theory are found in evolutionary biology, where by now thousands of papers have proved the fruitfulness of the approach (we refer to DR98 for a collection of survey papers, and [Si93] for a popularized account). In fact, questions of sex-ratio, and more generally of sex-allocation, even antedate any explicit formulation of evolutionary game theory. It was R.F. Fisher who used frequency-dependent selection to explain the prevalence of a 1:1 sex ratio [Fi30, and W.D. Hamilton who extended this type of thinking to make sense of other, odd sex ratios H67. Maynard Smith coined his concept of ESS to explain the prevalence of ritual fighting in intraspecific animal contests MS74]. The subtleties of such contests are still a favorite topic among the students of animal behaviour. More muted, but certainly not less widespread, conflicts arise on the issue of mate choice, parental investment, and parent-offspring conflicts (see e.g. [Tr74], Go95], MoPa97]). Social foraging is another field where the success of a given behaviour (scrounging, for instance) depends on its prevalence; so are dispersal and habitat selection. Communication (alarm calls, threat displays, sexual advertisement, gossip) with its possibilities of deceit is replete with game theoretical problems concerning bluff and honest signalling. Predators and their prey or parasites and their hosts offer examples of games between two populations, with the success of a trait depending on the state of the other population. Some 
strategic interactions are surprisingly sophisticated, considering the lowly level of the players: for instance, bacteria poisoning their neighbors or lizards attempting sneaky matings are engaged in rock-scissors-paper games [K02, [SL96].

Quite a few of these games turned out to have the same structure as games that had been studied by economists, usually under another name: the biologists' 'Hawk and Dove' and 'Battle of the Sexes' have the same structure as the economists' 'Chicken', resp. 'Buyer-Seller' game. A common language is slow in emerging, but by now hundreds of papers in economic theory use concepts from evolutionary games.

One zone of convergence for studies of animal behaviour and human societies is that of cooperation. Indeed, the theory of evolution and economic theory each have their own paradigm of selfishness, encapsulated in the slogans of the 'selfish gene' and the 'homo economicus', and both conflicting with widespread evidence of ultra-social behaviour. In ant and bee societies, the relatedness of individuals is so close that their genetic interests overlap and their communities can be viewed as 'superorganisms'. But in human societies, close cooperation can also occur between individuals who are unrelated. In many cases, such cooperation is based on reciprocation. The Prisoner's Dilemma game is the archetypical model displaying the conflict between selfishness and public good. It is a $2 \times 2$ game with two strategies - to cooperate or to defect. The latter is the dominating strategy, but the payoff, if both players defect, is less than the payoff if both cooperate. The repeated Prisoner's Dilemma is used to analyse reciprocating interactions A84. It admits a wealth of strategies but no strict equilibrium and no ESS FaW89. It has been investigated by extensive computer simulations of evolutionary game dynamics; see e.g. [NoS93]. The reciprocating strategy Tit for Tat and the unconditional strategy Always Defect are in bistable equilibrium and give rise to deep problems in coordination. The threat of punishment is another very efficient reason for the prevalence of cooperation [FG00]. Again, it leads to bistable equilibria, corresponding to behavioural norms; which of these norms eventually emerges depends on the history of the population Sk01.

Animal behaviour and experimental economics fuse in this area. Experimental economics has greatly flourished in the last few years [KR95: it often uses very simple games which can be analysed by means of evolutionary dynamics. Public goods games, for instance, with and without options of punishing the co-players for not contributing, lead to interesting two-stage games [SHN01. These are closely related to the Ultimatum game, where two players have to agree - without any opportunity of haggling - on how to split a sum offered by the experimenter or else forfeit that sum [GBS95], [NPS00]. These and other games display the limitations of 'rational' behaviour in humans [HB01]. Evolutionary game theory is not tied down by this assumption and hence allows us to suggest other explanations for 'irrational' solidarity and fairness.

4.8. The recurrence of 'Brownian' motion. The success of the replicator dynamics in the 1980's was due to its roots in evolutionary theory and 'population thinking', but the subsequent development has helped, interestingly, to re-appraise ideas from the (first) golden age of game theory, at the turn of the 1950s, as shown in [Ho00]. Two clusters of ideas stand out. One centers around the two dynamics introduced by G.W. Brown - one the 'fictitious play' approach leading to the BRdynamics (51), the other the BNN dynamics (65). In fact, if $f: \mathbf{R}_{+} \longrightarrow \mathbf{R}_{+}$is a 
continuous function satisfying $f(0)=0$ and $f(s)>0$ for $s>0$, and if $k_{i}(\mathbf{x})$ is given by (66), then

$$
\dot{x}_{i}=f\left(k_{i}\right)-x_{i} \sum_{j} f\left(k_{j}\right)
$$

is a common generalisation, as can be seen by using $f(s)=s^{\alpha}$ with $\alpha>0$. Indeed, for $\alpha=1$ one recovers the BNN dynamics and for $\alpha \rightarrow+\infty$ (after renormalisation) the BR dynamics. Interior ESS are globally asymptotically stable for (95). The same holds for the smoothed BR dynamics, as we have seen in section 3.4.

Intriguingly, the three proofs offered by John Nash for his equilibrium theorem (see [Na01]) are all related to a dynamic. These three proofs are based, respectively, on (a) the multivalued map $\mathbf{x} \rightarrow B R(\mathbf{x})$, and hence the BR-dynamics, (b) on the perturbed BR maps

$$
\mathbf{x} \mapsto \frac{\max \left(0,(A \mathbf{x})_{i}-\max _{k}(A \mathbf{x})_{k}+\varepsilon\right)}{\sum_{j} \max \left(0,(A \mathbf{x})_{j}-\max _{k}(A \mathbf{x})_{k}+\varepsilon\right)}
$$

and (c) on $\mathbf{x} \rightarrow \mathbf{f}(\mathbf{x})$ with $\mathbf{f}$ given by (67). On the other hand, our own proof in section 2.3 is based on the replicator dynamics.

Far from being a recent offshoot, evolutionary game dynamics is rooted firmly in the origins of game theory.

\section{ACKNOWLEDGEMENT}

We want to thank Carlos Alós-Ferrer, Ulrich Berger, Immanuel Bomze, Hannelore Brandt, Ross Cressman and two referees for their help. We acknowledge support from the Austrian Science Fund (P 15281 for JH, and WK 10008 for KS).

\section{REFERENCES}

[Ak79] E. Akin: The geometry of population genetics. Lecture Notes in Biomathematics 31. Springer, Berlin-Heidelberg-New York (1979) MR 80m:92007

[Ak93] E. Akin: The general topology of dynamical systems. Amer. Math. Soc., Providence (1993). MR 94f:58041

[AL84] E. Akin and V. Losert (1984): Evolutionary dynamics of zero-sum games, J. Math. Biology 20 (1984), 231-258. MR 86g:92024a

[AF00] C. Alós-Ferrer, Finite population dynamics and mixed equilibria, WP 0008, Department of Economics, University of Vienna, 2000. International Game Theory Review, forthcoming.

[AF01] C. Alós-Ferrer and A. Ania: Local Equilibria in Economic Games, Econ. Letters 70 (2001), 165-173. MR 2001j:91091

[AF02] C. Alós-Ferrer, A.B. Ania, The evolutionary logic of feeling small, WP 0216, Department of Economics, University of Vienna, 2002.

[AFS00] C. Alós-Ferrer, A.B. Ania and K.R. Schenk-Hoppé: An evolutionary model of Bertrand oligopoly. Games Econ. Behav. 33 (2000), 1-19. MR 2001i:91051

[ATW02] A.B. Ania, T. Tröger and A. Wambach: An evolutionary analysis of insurance markets with adverse selection, Games Econ. Behav. 40 (2002), 153-184.

[AuC84] J.P. Aubin, A. Cellina: Differential inclusions, Springer, Berlin (1984). MR 85j:49010 
[A84] R. Axelrod: The Evolution of Cooperation. New York: Basic Books (1984).

[BaS00] D. Balkenborg, K. Schlag: Evolutionarily stable sets, Int. J. Game Theory 29 (2000), 571-95. MR 2002c:91019

[Beg02] A. Beggs: On the convergence of reinforcement learning, Preprint.

[Ben99] M. Benaim: Dynamics of stochastic approximation algorithms. In: J. Azema et al.(eds.), Seminaire de probabilites XXXIII. Berlin: Springer. Lect. Notes Math. 1709, 1-68 (1999). MR 2001d:62081

[BenH99] M. Benaim and M.W. Hirsch: Mixed equilibria and dynamical systems arising from repeated games, Games Econ. Behav. 29 (1999), 36-72 MR 2000m:91020

[BenW00] M. Benaim and J. Weibull: Deterministic approximation of stochastic evolution in games, Preprint 2000, Econometrica. (To appear)

[Ber98] U. Berger: Best response dynamics and Nash dynamics for games, Dissertation, Univ. Vienna (1998).

[Ber01] U. Berger: Best response dynamics for role games, Int. J. Game Theory 30 (2001), 527-538. MR 2003c: 91008

[Ber02] U. Berger: A general model of best response adaptation. Preprint. 2002.

[Ber03] U. Berger: Fictitious play in $2 \times n$ games. Preprint. 2003.

[BerH02] U. Berger and J. Hofbauer: Irrational behavior in the Brown-von Neumann-Nash dynamics, Preprint 2002.

[B193] L.E. Blume: The statistical mechanics of strategic interaction, Games Econ. Behav. 5 (1993), 387-424. MR 94j:90063

[Bo83] I.M. Bomze: Lotka-Volterra equations and replicator dynamics: A two dimensional classification, Biol. Cybernetics 48 (1983), 201-211.

[Bo86] I.M. Bomze: Non-cooperative two-person games in biology: a classification Int. J. Game Theory 15 (1986), 31-57. MR 87g:90141

[Bo90] I.M. Bomze: Dynamical aspects of evolutionary stability, Monatshefte für Mathematik 110 (1990), 189-206 MR 92f:92023

[Bo91] I.M. Bomze: Cross entropy minimization in uninvadable states of complex populations, J. Math. Biol. 30 (1991), 73-87. MR 92j:92012

[Bo94] I.M. Bomze: Lotka-Volterra equation and replicator dynamics: new issues in classification, Biol. Cybernetics 72 (1994), 447-453.

[Bo98] I.M. Bomze: Uniform barriers and evolutionarily stable sets. In: W. Leinfellner, E. Köhler (eds.), Game Theory, Experience, Rationality, pp. 225-244. Kluwer, Dordrecht (1998), 225-244. MR 2001h:91020

[Bo02] I.M. Bomze: Regularity vs. degeneracy in dynamics, games, and optimization: a unified approach to different aspects, SIAM Review 44 (2002), 394-414.

[BoP89] I.M. Bomze, B.M. Pötscher: Game Theoretical Foundations of Evolutionary Stability, Lecture Notes in Economics and Math. Systems 324, Berlin: Springer (1989). MR 92i:92010

[BoB95] I.M. Bomze, R. Bürger: Stability by mutation in evolutionary games, Games and Economic Behaviour 11 (1995), 146-172. MR 97e:90122

[Bö00] T. Börgers: When Does Learning Lead to Nash Equilibrium?, in K. Inderfurth et al. (editors), Operations Research Proceedings 1999, Springer, Berlin (2000), 176-202.

[BöS97] T. Börgers, R. Sarin: Learning through reinforcement and replicator dynamics, J. Econ. Theory $7 \mathbf{7}$ (1997), 1-14. MR 98j:93087

[BH87] D.B. Brown and R.I.C. Hansel: Convergence to an evolutionarily stable strategy in the two-policy game, Amer. Naturalist 130 (1987), 929-940.

[Br49] G.W. Brown: Some notes on computation of games solutions, RAND report P-78 (1949).

[Br51] G.W. Brown: Iterative solution of games by fictitious play, in Activity Analysis of Production and Allocation, ed. T.C. Koopmans, New York, Wiley (1951), 374-376. MR 15:48e

[BrN50] G.W. Brown, J. von Neumann: Solutions of games by differential equations, Ann. Math. Studies 24 (1950), 73-79. MR 12:514a

[CaS92] A. Cabrales, J. Sobel: On the limit points of discrete selection dynamics, J. Econ. Theory 57 (1992), 407-419. MR 93g:90018

[Co92] S. Cowan: Dynamical systems arising from game theory, Dissertation (1992), Univ. California, Berkeley. 
[Cr90] R. Cressman: Strong stability and density-dependent evolutionarily stable strategies. J. Theor. Biology 145 (1990), 319-30. MR 91j:92010

[Cr92] R. Cressman: The stability concept of evolutionary game theory, Springer, Berlin (1992). MR 93j:92020

[Cr95] R. Cressman: Evolutionary game theory with two groups of individuals. Games Econ. Behav. 11 (1995), 237-253. MR 96m:92003

[Cr97] R. Cressman: Local stability for smooth selection dynamics for normal form games, Math. Social Sciences 34 (1997), 1-19. MR 98k:90167

[Cr03] R. Cressman: Evolutionary dynamics and extensive form games, Cambridge, Mass., MIT Press (2003).

[CrGH01] R. Cressman, J. Garay, J. Hofbauer: Evolutionary stability concepts for $N$-species frequency-dependent interactions, J. Theor. Biol. 211 (2001), 1-10.

[CrGW00] R. Cressman, A. Gaunersdorfer, J.F. Wen: Evolutionary and dynamic stability in symmetric evolutionary games with two independent decisions, Int. Game Theory Review 2 (2000), 67-81. MR 2001j:91019

[CrHH96] R. Cressman, J. Hofbauer, W.G.S. Hines: Evolutionary stability in strategic models of single-locus frequency-dependent viability selection. J. Math. Biol. 34 (1996), 707-733. MR 97j:92005

[CrVi97] R. Cressman, G. T. Vickers: Spatial and density effects in evolutionary game theory, J. Theor. Biol. 184 (1997), 359-369.

[DeS92] E. Dekel, S. Scotchmer: On the evolution of optimizing behavior, J. Econ. Theory 57 (1992), 392-346. MR 93f:90211

[DiLa96] U. Dieckmann, R. Law: The dynamical theory of coevolution, J. Math. Biol. 34 (1996), 579-612. MR 97m:92007

[DR98] L.A. Dugatkin and H.K. Reeve (eds.): Game Theory and Animal Behaviour, Oxford UP (1998)

[Du99] R. Durrett: Stochastic spatial models, SIAM Review 41 (1999) 677-718. MR 2000h:60086

[El93] G. Ellison: Learning, local interaction, and coordination, Econometrica 61 (1993), 1047-1071. MR 94g:90183

[ErR98] I. Erev and A. Roth: Predicting how people play games: Reinforcement learning in experimental games with unique, mixed strategy equilibrium, Amer. Econ. Review 88 (1998), 79-96.

[Es82] I. Eshel: Evolutionarily stable strategies and viability selection in Mendelian populations, Theor. Pop. Biol. 22 (1982), 204-217. MR 84f:92028

[Es83] I. Eshel: Evolutionary and continuous stability, J. Theor. Biol. 103 (1983), 99-111. MR 85a:92024

[Es96] I. Eshel: On the changing concept of evolutionary population stability as a reflection of a changing point of view in the quantitative theory of evolution, J. Math. Biol. 34 (1996), 485-510.

[EsAk83] I. Eshel, E. Akin: Coevolutionary instability of mixed Nash solutions, J. Math. Biology 18 (1983), 123-133. MR 85d:92023

[EsMS97] I. Eshel, U. Motro and E. Sansone, Continuous stability and evolutionary convergence, J. Theor. Biology 185 (1997), 333-343.

[EsSS98] I. Eshel, L. Samuelson and A. Shaked: Altruists, egoists and hooligans in a local interaction model, Amer. Econ. Review 88 (1998), 157-179.

[FaW89] J. Farrell and R. Ware: Evolutionary stability in the repeated prisoner's dilemma, Theor. Popul. Biol. 36 (1989), 161-166.

[FG00] E. Fehr and S. Gächter: Cooperation and punishment in public goods experiments, Amer. Econ. Review 90 (2000), 980-994.

[Fi79] P. Fife: Mathematical aspects of reacting and diffusing systems, Springer Lecture Notes in Biomathematics 28 (1979). MR 80g:35001

[Fi30] R.A. Fisher: The genetical theory of natural selection, Oxford: Clarendon Press (1930). MR 2002c:92013

[FoYo90] D. Foster and H. P. Young: Stochastic evolutionary game dynamics, Theor. Pop. Biol. 38 (1990), 219-232. MR 91h:92017 
[FoY98] D. Foster and H. P. Young: On the nonconvergence of fictitious play in coordination games, Games Econ. Behav. 25 (1998), 79-96. MR 99k:90217

[FrW84] M.I. Freidlin and A.D. Wentzel: Random perturbations of dynamical systems. New York: Springer (1984). MR 85a:60064

[Fr91] D. Friedman: Evolutionary games in economics, Econometrica 59 (1991), 637-66. MR 92b:90052

[FL98] D. Fudenberg and D. K. Levine: The theory of learning in games. MIT Press (1998). MR 99i:90121

[FT91] D. Fudenberg and J. Tirole: Game Theory. MIT Press (1991). MR 92m:90001

[GBS95] J. Gale, K. Binmore and L. Samuelson: Learning to be imperfect: the Ultimatum game, Games Econ. Behaviour 8 (1995), 56-90. MR 95k:90125

[GH03] B. Garay and J. Hofbauer, Robust permanence for ecological differential equations, minimax and discretizations. SIAM J. Math. Anal. 34 (2003), 1007-1093.

[Gau92] A. Gaunersdorfer: Time averages for heteroclinic attractors, SIAM J. Appl. Math 52 (1992), 1476-89. MR 93j:34068

[GaHS91] A. Gaunersdorfer, J. Hofbauer, K. Sigmund: On the dynamics of asymmetric games, Theor. Pop. Biol. 39 (1991), 345-357. MR 92d:90136

[GaH95] A. Gaunersdorfer, J. Hofbauer: Fictitious play, Shapley polygons and the replicator equation, Games Econ. Behav. 11 (1995), 279-303. MR 96j:90096

[Ge02] S.A.H. Geritz, M. Gyllenberg, F.J.A. Jacobs, K. Parvinen: Invasion dynamics and attractor inheritance, J. Math. Biol. 44 (2002), 548-560. MR 2003d:92021

[GiM91] I. Gilboa, A. Matsui: Social stability and equilibrium, Econometrica 59 (1991), 859-867.

[Gi00] H. Gintis: Game theory evolving, Princeton UP (2000).

[Go95] Godfray, H.C.J. (1995) Evolutionary theory of parent- offspring conflict, Nature 376, 133-138

[H67] W.D. Hamilton: Extraordinary sex ratios, Science 156 (1967), 477-488.

[Ha96] P. Hammerstein: Darwinian adaptation, population genetics and the streetcar theory of evolution, J. Math. Biol. 34 (1996), 511-532.

[HaS94] P. Hammerstein, R. Selten: Game theory and evolutionary biology, in R.J. Aumann, S. Hart (eds.): Handbook of Game Theory II, Amsterdam: North-Holland (1994), 931-993.

[Har73] J.C. Harsanyi: Oddness of the number of equilibrium points: a new proof, Int. J. Game Theory 2 (1973), 235-250. MR 58:26059

[HaSe88] J.C. Harsanyi, R. Selten: A General Theory of Equilibrium Selection in Games, MIT Press (1988). MR 89j:90285

[Har98] C. Harris: On the rate of convergence of continuous- time fictitious play, Games Econ. Behav. 22 (1998), 238-259. MR 99d:90147

[HaM01] S. Hart, A. Mas-Colell: A general class of adaptive strategies, J. Econ. Theory 98 (2001), 26-54. MR 2003b:91017

[HB01] J. Henrich, R. Boyd, S. Bowles, C. Camerer, E. Fehr, H. Gintis and R. McElreath: In search of homo economicus: behavioral experiments in 15 small-scale societies, Amer. Econ. Rev. 91 (2001), 73-78

[Hi87] W.G.S. Hines: Evolutionary stable strategies: a review of basic theory. Theor. Pop. Biol. 31 (1987), 195-272. MR 88f:92033

[Hi94] W.G.S. Hines: ESS modelling of diploid populations I and II. J. Appl. Prob. 26 (1994), 341-376. MR 95g:92009a

[Hi88] M. W. Hirsch: Systems of differential equations which are competitive or cooperative: III. Competing species, Nonlinearity 1 (1988), 51-71. MR 90d:58070

[Ho81] J. Hofbauer: On the occurrence of limit cycles in the Volterra-Lotka equation, Nonlinear Analysis 5 (1981), 1003-1007. MR 83c:92063

[Ho84] J. Hofbauer: A difference equation model for the hypercycle, SIAM J. Appl. Math. 44 (1984), 762-772. MR 85m:58153

[Ho95a] J. Hofbauer: Stability for the best response dynamics, Preprint.

[Ho95b] J. Hofbauer: Imitation dynamics for games, Preprint.

[Ho96] J. Hofbauer: Evolutionary dynamics for bimatrix games: a Hamiltonian system?, J. Math. Biol. 34 (1996), 675-688. MR 97h:92011 
[Ho97] J. Hofbauer: Equilibrium selection via travelling waves. In: Werner Leinfellner and Eckehart Köhler (eds.), Vienna Circle Institute Yearbook 5/1997, Game Theory, Experience, Rationality. Foundations of Social Sciences, Economics and Ethics. Harsanyi. Dordrecht-Boston-London: Kluwer (1998), 245-259. MR 2001f:91018

[Ho99] J. Hofbauer: The spatially dominant equilibrium of a game. Annals of Operations Research 89 (1999) 233-251. MR 2000g:91008

[Ho00] J. Hofbauer: From Nash and Brown to Maynard Smith: equilibria, dynamics and ESS, Selection 1 (2000), 81-88.

[HoH02] J. Hofbauer and E. Hopkins: Learning in perturbed asymmetric games. Preprint. 2002.

[HoHV97] J. Hofbauer, V. Hutson and G.T. Vickers, Travelling waves for games in economics and biology, Nonlinear Analysis 30 (1997) 1235-1244.

[HoOR03] J. Hofbauer, J. Oechssler and F. Riedel: Continuous and global stability in innovative evolutionary dynamics. Preprint. 2003.

[HoSa01] J. Hofbauer, W.H. Sandholm: Evolution and learning in games with randomly perturbed payoffs, Preprint, 2001.

[HoSa02] J. Hofbauer, W.H. Sandholm: On the global convergence of stochastic fictitious play, Econometrica 70 (2002), 2265-94.

[HoSc00] J. Hofbauer, K. Schlag: Sophisticated imitation in cyclic games, J. Evolutionary Economics 10 (2000), 523-543.

[HoSS79] J. Hofbauer, P. Schuster, K. Sigmund: A note on evolutionary stable strategies and game dynamics, J. Theor. Biology 81 (1979), 609-612. MR 81d:92015

[HoSS82] J. Hofbauer, P. Schuster, K. Sigmund: Game dynamics in Mendelian populations. Biol. Cybern. 43 (1982), 51-57. MR 83j:92069

[HoS88] J. Hofbauer, K. Sigmund: The Theory of Evolution and Dynamical Systems, Cambridge UP (1988). MR 91h:92019

[HoS90] J. Hofbauer, K. Sigmund: Adaptive dynamics and evolutionary stability. Appl. Math. Letters 3 (1990), 75-79. MR 91h:92018

[HoSi98] J. Hofbauer, K. Sigmund: Evolutionary Games and Population Dynamics, Cambridge UP (1998). MR 99h:92027

[HSo94] J. Hofbauer, J. W.-H. So: Multiple limit cycles in three dimensional Lotka-Volterra equations. Appl. Math. Letters 7 (1994), 65-70. MR 96g:34063

[HoSo02] J. Hofbauer and S. Sorin: Best response dynamics for continuous zero-sum games. Preprint, 2002.

[HoSw96] J. Hofbauer, J. Swinkels: A universal Shapley example. Preprint.

[HoW96] J. Hofbauer, J. W. Weibull: Evolutionary selection against dominated strategies, J. Econ. Theory 71 (1996), 558-573. MR 97k:92012

[Hop99a] E. Hopkins: Learning, matching, aggregation, Games Econ. Behav. 26 (1999), 79110. MR 2000c:91004

[Hop99b] E. Hopkins: A note on best response dynamics, Games Econ. Behav. 29 (1999), 138-50. MR 2000j:91017

[Hop02] E. Hopkins: Two Competing Models of How People Learn in Games, Econometrica 70 (2002), 2141-2166.

[HopP02] E. Hopkins and M. Posch: Attainability of boundary points under reinforcement learning, Preprint.

[HuVi92] V. Hutson, G.T. Vickers: Travelling waves and dominance of ESS's, J. Math. Biol. 30 (1992), 457-471. MR 93g:90112

[JJS99] H.J. Jacobsen, M. Jensen and B. Sloth: On the structural difference between the evolutionary approach of Young and that of KMR. Preprint.

[KR95] J.H. Kagel and A.E. Roth: Handbook of Experimental Economics, Princeton UP (1995).

[Ka97] M. Kandori: Evolutionary Game Theory in Economics, in D. M. Kreps and K. F. Wallis (eds.), Advances in Economics and Econometrics: Theory and Applications, I, Cambridge UP (1997).

[KaMR93] M. Kandori, G.J. Mailath and R. Rob: Learning, mutation, and long run equilibria in games, Econometrica 61 (1993), 29-56. MR 93m:90129

[KaR95] M. Kandori and R. Rob: Evolution of equilibria in the long run: a general theory and applications, J. Econ. Theory 65 (1995), 383-414. MR 96b:90130 
[K02] B. Kerr, M.A. Riley, M.W. Feldman and B.J.M. Bohannan: Local dispersal promotes biodiversity in a real-life game of rock-paper-scissors, Nature 418 (2002), 171-174.

[Kos02] M. Kosfeld: Stochastic strategy adjustment in coordination games, Economic Theory 20 (2002), 321-339. MR 2003f:91005

[Kr92] V. Krishna: Learning in games with strategic complementarities, Preprint.

[KrS98] V. Krishna, T. Sjöström: On the convergence of fictitious play, Math. Oper. Res. 23 (1998), 479-511. MR 99i:90120

[LaTW01] J.F. Laslier, R. Topol, B. Walliser: A behavioral learning process in games, Games Econ. Behav. 37 (2001), 340-366. MR 2002k:91040

[Le94] R.J. Leonard: Reading Cournot, reading Nash: The creation and stabilisation of the Nash equilibrium, The Economic Journal 104 (1994), 492-511.

[Le84] S. Lessard: Evolutionary dynamics in frequency dependent two-phenotypes models, Theor. Pop. Biol. 25 (1984), 210-234.

[Le90] S. Lessard: Evolutionary stability: one concept, several meanings. Theor. Pop. Biol. 37 (1990), 159-70. MR 90m:92042

[LoA83] V. Losert and E. Akin: Dynamics of games and genes: Discrete versus continuous time, J. Math. Biology 17 (1983), 241-251. MR 85a:92019

[Lu02] Z. Lu, Y. Luo, Three limit cycles for a three-dimensional Lotka-Volterra competitive system with a heteroclinic cycle, Computer Math. Appl., to appear.

[Mai98] G. Mailath: Do people play Nash equilibrium? Lessons from evolutionary game theory, J. Economic Literature 36 (1998), 1347-1374.

[Ma92] A. Matsui: Best response dynamics and socially stable strategies, J. Econ. Theory 57 (1992), 343-362. MR 93g:90110

[MS74] J. Maynard Smith: The theory of games and the evolution of animal conflicts, J. Theor. Biol. 47 (1974), 209-221. MR56:2475

[MS81] J. Maynard Smith: Will a sexual population evolve to an ESS? Amer. Naturalist 177 (1981), 1015-1018.

[MS82] J. Maynard Smith: Evolution and the Theory of Games. Cambridge Univ. Press (1982).

[MS88] J. Maynard Smith: Can a mixed strategy be stable in a finite population?, J. Theor. Biol. 130 (1988), 247-251. MR 89d:92047

[Mayr70] E. Mayr: Populations, species, and evolution. Harvard Univ. Press (1970).

[Me01]

G. Meszéna, È. Kisdi, U. Dieckmann, S.A.H. Geritz, J.A.J. Metz: Evolutionary optimization models and matrix games in the unified perspective of adaptive dynamics, Selection 2 (2001), 193-210.

[Me96] J.A.J. Metz, S.A.H. Geritz, G. Meszéna, F.J.A. Jacobs, J.S. van Heerwarden: Adaptive dynamics: a geometrical study of the consequences of nearly faithful replication, in S.J. Van Strien, S.M. Verduyn Lunel (eds.) Stochastic and spatial structures of dynamical systems, Amsterdam: North Holland (1996), 183-231. MR 2001b:37006

[MoPa97] D. W. Mock and G.A. Parker: The evolution of sibling rivalry. Oxford UP (1997).

[MoSh96a] D. Monderer, L. Shapley: Potential games, Games Econ. Behav. 14 (1996), 124143. MR 97g:90183

[MoSh96b] D. Monderer, L. Shapley: Fictitious play property for games with identical interests, J. Econ. Theory 68 (1996), 258-265. MR 96m:90144

[Mor00] S. Morris, Contagion, Review of Economic Studies 67 (2000), 57-78. MR 2001j:91020

[Nac90] J. Nachbar: "Evolutionary" selection dynamics in games: convergence and limit properties, Int. J. Game Theory 19 (1990), 59-89. MR 91c:90127

[Na51] J. Nash: Non-cooperative games, Ann. Math. 54 (1951), 287-295. MR 13:261g

[Na96] J. Nash: Essays on Game Theory, Elgar, Cheltenham (1996).

[Na01] J. Nash: The essential John Nash, Eds: H.W. Kuhn and S. Nasar, Princeton UP (2002). MR 2002k:01044

[NM47] J. von Neumann, O. Morgenstern: Theory of Games and Economic Behaviour, Princeton UP (1947). MR 9:50f

[Ni59] H. Nikaido: Stability of equilibrium by the Brown-von Neumann differential equation, Econometrica 27 (1959), 654-671. MR 22:6600 
[No90] M.A. Nowak: An evolutionarily stable strategy may be inaccessible, J. Theor. Biol. 142 (1990), 237-241. MR 91e:92016

[NoBoMay94] M.A. Nowak, S. Bonhoeffer and R.M. May: More spatial games, Int. J. Bif. Chaos 4 (1994), 33-56. MR 95a:90213

[NoMay92] M.A. Nowak and R.M. May: Evolutionary games and spatial chaos, Nature 359 (1992), 826-829

[NoMay93] M.A. Nowak and R.M. May: The spatial dilemmas of evolution, Int. J. Bifur. Chaos Appl. Sci. Engrg. 3 (1993), 35-78. MR 94c:92014

[NoS93] M.A. Nowak and K. Sigmund: Chaos and the evolution of cooperation, Proc. Nat. Acad. Science USA 90 (1993), 5091-94.

[NPS00] M.A. Nowak, K.P. Page and K. Sigmund: Fairness versus reason in the ultimatum game, Science 289 (2000), 1773-1775.

[NS97] G. Nöldeke and L. Samuelson: A dynamic model of equilibrium selection in signaling markets. J. Econ. Theory 73 (1997), 118-156.

[Oe97] J. Oechssler: An evolutionary interpretation of mixed-strategy equilibria, Games Econ. Behav. 21 (1997), 203-237. MR 98m:90194

[OR01] J. Oechssler and F. Riedel: Evolutionary dyamics on infinite strategy spaces, Economic Theory 17 (2001), 141-162. MR 2002c:91020

[OR02] J. Oechssler and F. Riedel: On the dynamic foundation of evolutionary stability in continuous models, J. Econ. Theory 107 (2002), 223-252.

[P197] M. Plank: Some qualitative differences between the replicator dynamics of two player and $n$ player games, Nonlinear Analysis 30 (1997), 1411-1417.

[Po97] M. Posch: Cycling in a stochastic learning algorithm for normal form games, J. Evol. Economics 7 (1997), 193-207.

[RS01] P.W. Rhode and M. Stegemann: Evolution through imitation: The case of duopoly, Int. J. Industrial Organization 19 (2001), 415-454.

[RiW95] K. Ritzberger, J. W. Weibull: Evolutionary selection in normal-form games, Econometrica 63 (1995), 1371-1399. MR 96h:90147

[Ro51] J. Robinson: An iterative method of solving a game, Ann. Math. (2) 54 (1951), 296-301. MR 13:261e

[Ro71] J. Rosenmüller: Über Periodizitätseigenschaften spieltheoretischer Lernprozesse, Z. Wahrscheinlichkeitstheorie Verw. Geb. 17 (1971), 259-308. MR 44:6351

[Ru99] A. Rustichini: Optimal properties of stimulus- response learning models. Games Econ. Behav. 29 (1999), 244-273. MR 2000m:91024

[Sa97] L. Samuelson: Evolutionary games and equilibrium selection, Cambridge, MIT Press (1997). MR 98e:90003

[SaZ92] L. Samuelson, J. Zhang: Evolutionary stability in asymmetric games, J. Econ. Theory 57 (1992), 363-391. MR 93f:90188

[Sa98] W.H. Sandholm: Simple and clever decision rules for a model of evolution, Econ. Lett. 61 (1998), 165-170. MR 99i:92011

[Sa01] W.H. Sandholm: Potential games with continuous player sets, J. Econ. Theory $\mathbf{9 7}$ (2001), 81-108. MR 2002b:91005

[Sa02] W.H. Sandholm: Excess payoff dynamics, potential dynamics and stable games. Preprint.

[Sat02] Y. Sato, E. Akiyama, J. Doyne Farmer: Chaos in learning a simple two-person game, Proc. Nat. Acad. Sci. 99 (2002), 4748-4751. MR 2003e:91006

[S88] M.E. Schaffer: Evolutionarily stable strategies for a finite population and a variable contest size, J. Theor. Biol. 132 (1988), 469-478. MR 89j:92071

[S89] M.E. Schaffer: Are profit-maximisers the best survivors?, J. Econ. Behav. Org. 12 (1989), 29-45.

[Sc97] K.H. Schlag: Why imitate, and if so, how? A boundedly rational approach to multi-armed bandits, J. Econ. Theory 78 (1998), 130-156. MR 99h:90121

[Sch01] S. Schreiber: Urn models, replicator processes and random genetic drift, SIAM J. Applied Math 61 (2001), 2148-2167. MR 2002h:92019

[ScS83] P. Schuster, K. Sigmund: Replicator dynamics, J. Theor. Biology 100 (1983), 533538. MR 85i:92006

[Se00] A. Sela: Fictitious play in $2 \times 3$ games, Games Econ. Behav. 31 (2000), 152-162. MR 2000m:91006 
[Se80] R. Selten: A note on evolutionarily stable strategies in asymmetrical animal conflicts, J. Theor. Biology 84 (1980), 93-101. MR 81d:92016

[Se98] R. Sethi: Strategy-specific barriers to learning and nonmonotonic selection dynamics, Games Econ. Behav. 23 (1998), 284-309. MR 99b:90168

[Sh64] L. Shapley: Some topics in two-person games, Ann. Math. Studies 5 (1964), 1-28. MR 33:7140

[Si87] K. Sigmund: Game dynamics, mixed strategies and gradient systems, Theor. Pop. Biol. 32 (1987), 114-126. MR 88j:92065

[Si93] K. Sigmund: Games of Life. Penguin, Harmondsworth. 1993.

[SHN01] K. Sigmund, C. Hauert and M.A. Nowak: Reward and punishment in minigames, Proc. Nat. Acad. Sci. 98 (2001), 10757-10762.

[SL96] B. Sinervo and C.M. Lively: The rock-paper-scissors game and the evolution of alternative male strategies, Nature 380 (1996), 240-243.

[Sk90] B. Skyrms: The Dynamics of Rational Deliberation. Harvard UP (1990). MR 92d:90008

[Sk01] B. Skyrms: The stag hunt, Proceedings and Addresses of the American Philosophical Association. To appear.

[Sm95] H. Smith: Monotone Dynamical Systems: An Introduction to the Theory of Competitive and Cooperative Systems. Amer. Math. Soc. Math. Surveys and Monographs, Vol. 41 (1995). MR 96c:34002

[Sw92] J. Swinkels: Evolutionary stability with equilibrium entrants, J. Econ. Theory $\mathbf{5 7}$ (1992), 306-32. MR 93k:90125

[Sw93] J. Swinkels: Adjustment dynamics and rational play in games, Games Econ. Behav. 5 (1993), 455-84. MR 94b:90112

[TaKi91] T. Takada, J. Kigami: The dynamical attainability of ESS in evolutionary games, J. Math. Biol. 29 (1991), 513-529. MR 92f:92030

[Tay89] P.D. Taylor: Evolutionary stability in one-parameter models under weak selection. Theor. Pop. Biol. 36 (1989), 125-143. MR 90k:92030

[TaJ78] P.D. Taylor, L. Jonker: Evolutionarily stable strategies and game dynamics, Math. Biosciences 40 (1978), 145-156. MR 58:9351

[Th85] B. Thomas: On evolutionarily stable sets, J. Math. Biol. 22 (1985), 105-115. MR 86m:92039

[Tr74] R.L. Trivers: Parent-offspring conflict, Amer. Zool. 14 (1974), 249-264.

[vD91] E. van Damme: Stability and perfection of Nash equilibria, 2nd edition, Springer, Berlin (1991). MR 95f:90001

[V96] F. Vega-Redondo: Evolution, games, and economic theory. Oxford UP (1996).

[V97] F. Vega-Redondo: The evolution of Walrasian behavior. Econometrica 65 (1997), 375-384.

[Vi89] G.T. Vickers: Spatial patterns and ESS's, J. Theor. Biology 140 (1989) 129-135. MR 90j:92016

[We95] J. Weibull: Evolutionary Game Theory. MIT Press, Cambridge, Mass. (1995). MR 96f:90004

[Wei91] F. Weissing: Evolutionary stability and dynamic stability in a class of evolutionary normal form games. In R. Selten (ed.) Game Equilibrium Models I, Berlin, Springer (1991), 29-97.

[Wei96] F. Weissing: Genetic versus phenotypic models of selection: can genetics be neglected in a long-term perspective?, J. Math. Biol. 34 (1996), 533-555.

[Y93] H.P. Young: The evolution of conventions. Econometrica 61 (1993), 57-84. MR 93j:92038

[Y98] H.P. Young: Individual strategy and social structure, Princeton UP (1998).

[Ze80] E.C. Zeeman: Population dynamics from game theory. In: Global Theory of Dynamical Systems. Springer Lecture Notes in Mathematics 819 (1980). MR 82e:58076

[Ze93] M.L. Zeeman: Hopf bifurcations in competitive three dimensional Lotka-Volterra systems, Dynam. Stability Systems 8 (1993), 189-217. MR 94j:34044

[ZZ02] E.C. Zeeman, M.L. Zeeman: An n-dimensional competitive Lotka-Volterra system is generically determined by its edges, Nonlinearity 15 (2002), 2019-2032.

[ZZ03] E.C. Zeeman, M.L. Zeeman: From local to global behavior in competitive LotkaVolterra systems, Trans. Amer. Math. Soc. 355 (2003), 713-734. 
(Hofbauer) Institut für Mathematik, Universität Wien, Strudlhofgasse 4, A-1090 VIENNA, AUstria

E-mail address: Josef.Hofbauer@univie.ac.at

(Sigmund) Institut für Mathematik, Universität Wien, Strudlhofgasse 4, A-1090 ViENNA, AUSTRIa

E-mail address: Karl.Sigmund@univie.ac.at

(Sigmund) IIASA, A-2361 Laxenburg, Austria 